\title{
Algorithms on Minimizing the Maximum Sensor Movement for Barrier Coverage of a Linear Domain
}

\author{
Danny Z. Chen* $\quad$ Yan $\mathrm{Gu}^{\dagger} \quad$ Jian $\mathrm{Li}^{\ddagger} \quad$ Haitao Wang ${ }^{\S}$
}

\begin{abstract}
In this paper, we study the problem of moving $n$ sensors on a line to form a barrier coverage of a specified segment of the line such that the maximum moving distance of the sensors is minimized. Previously, it was an open question whether this problem on sensors with arbitrary sensing ranges is solvable in polynomial time. We settle this open question positively by giving an $O\left(n^{2} \log n\right)$ time algorithm. For the special case when all sensors have the same-size sensing range, the previously best solution takes $O\left(n^{2}\right)$ time. We present an $O(n \log n)$ time algorithm for this case; further, if all sensors are initially located on the coverage segment, our algorithm takes $O(n)$ time. Also, we extend our techniques to the cycle version of the problem where the barrier coverage is for a simple cycle and the sensors are allowed to move only along the cycle. For sensors with the same-size sensing range, we solve the cycle version in $O(n)$ time, improving the previously best $O\left(n^{2}\right)$ time solution.
\end{abstract}

\section{Introduction}

A Wireless Sensor Network (WSN) uses a large number of sensors to monitor some surrounding environmental phenomena [1]. Each sensor is equipped with a sensing device with limited batterysupplied energy. The sensors process data obtained and forward the data to a base station. Intrusion detection and border surveillance constitute a major application category for WSNs. A main goal of these applications is to detect intruders as they cross the boundary of a region or domain. For example, research efforts were made to extend the scalability of WSNs to the monitoring of international borders [11, 12]. Unlike the traditional full coverage [14, 19, 20] which requires an entire target region to be covered by the sensors, the barrier coverage [2, 3, 9, 10, 12] only seeks to cover the perimeter of the region to ensure that any intruders are detected as they cross the region border. Since barrier coverage requires fewer sensors, it is often preferable to full coverage. Because sensors have limited battery-supplied energy, it is desired to minimize their movements. In this paper, we study a one-dimensional barrier coverage problem where the barrier is for a (finite) line segment and the sensors are initially located on the line containing the barrier segment and allowed to move on the line. As discussed in the previous work [9, 10, 17] and shown in this paper, barrier coverage even for 1-D domains poses some challenging algorithmic issues. Also, our 1-D solutions may be used in solving more general problems. For example, if the barrier is sought for a simple polygon, then we may consider each of its edges separately and apply our algorithms to each edge.

In our problem, each sensor has a sensing range (or range for short) and we want to move the sensors to form a coverage for the barrier such that the maximum sensor movement is minimized.

\footnotetext{
${ }^{*}$ Department of Computer Science and Engineering, University of Notre Dame, Notre Dame, IN 46556, USA. E-mail: dchen@nd.edu. The research of Chen was supported in part by NSF under Grants CCF-0916606 and CCF1217906.

${ }^{\dagger}$ Department of Computer Science and Technology, Tsinghua University, Beijing 100084, China. E-mail: henryy321@gmail.com.

${ }^{\ddagger}$ Institute for Interdisciplinary Information Sciences (IIIS), Tsinghua University, Beijing 100084, China. E-mail: lijian83@mail.tsinghua.edu.cn.

${ }^{\S}$ Corresponding author. Department of Computer Science, Utah State University, Logan, UT 84322, USA. E-mail: haitao. wang@usu.edu. This work was partially done while the author was visiting IIIS at Tsinghua University.
} 
We present efficient algorithms for this problem, improving the previous work and settling an open question. Also, we extend our techniques to the cycle version where the barrier is for a simple cycle and the sensors are allowed to move only along the cycle.

\subsection{Problem Definitions, Previous Work, and Our Results}

Denote by $B=[0, L]$ the barrier that is a line segment from $x=0$ to $x=L>0$ on the $x$-axis. A set $S=\left\{s_{1}, s_{2}, \ldots, s_{n}\right\}$ of $n$ mobile sensors is initially located on the $x$-axis. Each sensor $s_{i} \in S$ has a range $r_{i}>0$ and is located at the coordinate $x_{i}$ of the $x$-axis. We assume $x_{1} \leq x_{2} \leq \cdots \leq x_{n}$. If a sensor $s_{i}$ is at the position $x^{\prime}$, then we say $s_{i}$ covers the interval $\left[x^{\prime}-r_{i}, x^{\prime}+r_{i}\right]$, called the covering interval of $s_{i}$. Our problem is to find a set of destinations on the $x$-axis, $\left\{y_{1}, y_{2}, \ldots, y_{n}\right\}$, for the sensors (i.e., for each $s_{i} \in S$, move $s_{i}$ from $x_{i}$ to $y_{i}$ ) such that each point on the barrier $B$ is covered by at least one sensor and the maximum moving distance of the sensors (i.e., $\max _{1 \leq i \leq n}\left\{\left|x_{i}-y_{i}\right|\right\}$ ) is minimized. We call this problem the barrier coverage on a line segment, denoted by BCLS. We assume $2 \cdot \sum_{i=1}^{n} r_{i} \geq L$ (otherwise, a barrier coverage for $B$ is not possible).

The decision version of BCLS is defined as follows. Given a value $\lambda \geq 0$, determine whether there is a feasible solution in which each point of $B$ is covered by at least one sensor and the moving distance of each sensor is at most $\lambda$. The decision version characterizes a problem model in which the sensors have a limited energy and we want to know whether their energy is sufficient to move and form a barrier coverage.

If the ranges of all sensors are the same (i.e., the $r_{i}$ 's are all equal), then we call it the uniform case of BCLS. When the sensors have arbitrary ranges, we call it the general case.

The BCLS problem has been studied before. The uniform case has been solved in $O\left(n^{2}\right)$ time [9]. An $O(n)$ time algorithm is also given in [9] for the decision version of the uniform case. However, it has been open whether the general case is solvable in polynomial time [9].

In this paper, we settle the open problem on the general BCLS, presenting an $O\left(n^{2} \log n\right)$ time algorithm for it. We also solve the decision version of the general BCLS in $O(n \log n)$ time. Since this is a basic problem on sensors and intervals and our algorithm is the first-known polynomial time solution for it, we expect our results and techniques to be useful for other related problems. Further, for the uniform case, we derive an $O(n \log n)$ time algorithm, improving the previous $O\left(n^{2}\right)$ time solution [9]; if all sensors are initially on $B$, our algorithm runs in $O(n)$ time.

In addition, we consider the simple cycle barrier coverage where the barrier is represented as a simple cycle and the $n$ sensors are initially on the cycle and are allowed to move only along the cycle. The goal is to move the sensors to form a barrier coverage and minimize the maximum sensor movement. If all sensors have the same range, Mehrandish [16] gave an $O\left(n^{2}\right)$ time algorithm, and we present an $O(n)$ time solution in this paper.

\subsection{Related Work}

Besides the results mentioned above, an $O(n)$ time 2-approximation algorithm for the uniform BCLS was also given in [9] and a variation of the decision version of the general BCLS, where one sensor is required to be in a given position in the final solution, is shown to be NP-hard [9]; however, it is not known whether the general BCLS is NP-hard. Further, in the special case where the order preserving property holds, i.e., $y_{1} \leq y_{2} \cdots \leq y_{n}$ holds in an optimal solution, polynomial time algorithms exist for the general BCLS [9]. Additional results were also given in [9] for the case $2 \cdot \sum_{i=1}^{n} r_{i}<L$ (although in this case $B$ cannot be entirely covered). 
Mehrandish et al. [16, 17] also considered the line segment barrier, but unlike the BCLS problem, they intended to use the minimum number of sensors to form a barrier coverage, which they proved to be NP-hard. But, if all sensors have the same range, polynomial time algorithms were possible [16, 17]. Another study of the line segment barrier [10] aimed to minimize the sum of the moving distances of all sensors; this problem is NP-hard [10], but is solvable in polynomial time when all sensors have the same range [10]. In addition, Li et al. [13] considers the linear coverage problem which aims to set an energy for each sensor to form a coverage such that the cost of all sensors is minimized. There [13, the sensors are not allowed to move, and the more energy a sensor has, the larger the covering range of the sensor and the larger the cost of the sensor.

Bhattacharya et al. [2] studied a 2-D barrier coverage problem in which the barrier is a circle and the sensors, initially located inside the circle, are moved to the circle to form a coverage such that the maximum sensor movement is minimized; the ranges of the sensors are not explicitly specified but the destinations of the sensors are required to form a regular $n$-gon on the circle. Subsequent improvements of the results in [2] have been made [5, 18. In addition, Bhattacharya et al. [2] presented some results on the corresponding min-sum problem version (minimizing the sum of the moving distances of all sensors); further improvement was also given in [5, 18.

Some other barrier coverage problems have been studied. For example, Kumar et al. [12] proposed algorithms for determining whether a region is barrier covered after the sensors are deployed. They considered both the deterministic version (the sensors are deployed deterministically) and the randomized version (the sensors are deployed randomly), and aimed to determine a barrier coverage with high probability. Chen et al. [3] introduced a local barrier coverage problem in which individual sensors determine the barrier coverage locally.

\subsection{An Overview of Our Approaches}

For any problem we consider, let $\lambda^{*}$ denote the maximum sensor movement in an optimal solution.

For the uniform BCLS, as shown in [9], a useful property is that there always exists an order preserving optimal solution, i.e., the order of the sensors in the optimal solution is the same as that in the input. Based on this property, the previous $O\left(n^{2}\right)$ time algorithm [9] covers $B$ from left to right; in each step, it picks the next sensor and re-balances the current maximum sensor movement. In this paper, we take a very different approach. With the order preserving property, we determine a set $\Lambda$ of candidate values for $\lambda^{*}$ with $\lambda^{*} \in \Lambda$. Consequently, by using the decision algorithm, we can find $\lambda^{*}$ in $\Lambda$. But, this approach may be inefficient since $|\Lambda|=\Theta\left(n^{2}\right)$. To reduce the running time, our strategy is not to compute the set $\Lambda$ explicitly. Instead, we compute an element in $\Lambda$ whenever we need it. A possible attempt would be to first find a sorted order for the elements of $\Lambda$ or (implicitly) sort the elements of $\Lambda$, and then obtain $\lambda^{*}$ by binary search. However, it seems not easy to (implicitly) sort the elements of $\Lambda$. Instead, based on several new observations, we manage to find a way to partition the elements of $\Lambda$ into $n$ sorted lists, each list containing $O(n)$ elements. Next, by using a technique called binary search on sorted arrays [6], we are able to find $\lambda^{*}$ in $\Lambda$ in $O(n \log n)$ time. For the special case when all sensors are initially located on $B$, a key observation we make is that $\lambda^{*}$ is precisely the maximum value of the candidate set $\Lambda$. Although $\Lambda=\Theta\left(n^{2}\right)$, based on new observations, we show that its maximum value can be computed in $O(n)$ time.

For the general BCLS, as indicated in [9], the order preserving property no longer holds. Consequently, our approach for the uniform case does not work. The main difficulty of this case is that we do not know the order of the sensors appeared in an optimal solution. Due to this difficulty, no polynomial time algorithm was known before for the general BCLS. To solve this problem, we first develop a greedy algorithm for the decision version of the general BCLS. After $O(n \log n)$ time 
preprocessing, our decision algorithm takes $O(n)$ time for any value $\lambda$. If $\lambda \geq \lambda^{*}$, implying that there exists a feasible solution, then our decision algorithm can determine the order of sensors in a feasible solution for covering $B$. For the general BCLS, we seek to simulate the behavior of the decision algorithm on $\lambda=\lambda^{*}$. Although we do not know the value $\lambda^{*}$, our algorithm determines the same sensor order as it would be obtained by the decision algorithm on the value $\lambda=\lambda^{*}$. To this end, each step of the algorithm uses our decision algorithm as a decision procedure. The idea is somewhat similar to parametric search [7, 15], and here we "parameterize" our decision algorithm. However, we should point out a few differences. First, unlike the typical parametric search [7, 15], our approach does not involve any parallel scheme and is practical. Second, normally, if a problem can be solved by parametric search, then there also exist other (simpler) polynomial time algorithms for the problem although they might be less efficient than the parametric search solution (e.g., the slope selection problem [8]). In contrast, for our general BCLS problem, so far we have not found any other (even straightforward) polynomial time algorithm.

In addition, our $O(n)$ time algorithm for the simple cycle barrier coverage is a generalization of our approach for the special case of the uniform BCLS when all sensors are initially located on $B$.

For ease of exposition, we assume that initially no two sensors are located at the same position (i.e., $x_{i} \neq x_{j}$ for any $i \neq j$ ), and the covering intervals of any two different sensors do not share a common endpoint. Our algorithms can be easily generalized to the general situation.

The rest of the paper is organized as follows. In Section 2, we describe our algorithms for the general BCLS. In Section 3, we present our algorithms for the uniform BCLS. Our results for the simple cycle barrier coverage are discussed in Section 4. Section 5 finally concludes the paper.

\section{The General Case of BCLS}

In this section, we present our algorithms for the general BCLS problem. Previously, it was an open problem whether the general BCLS can be solved in polynomial time. The main difficulty is that we do not know the order of the sensors in an optimal solution. Our main effort is for resolving this difficulty, and we derive an $O\left(n^{2} \log n\right)$ time algorithm for the general BCLS.

We first give our algorithm for the decision version (in Section 2.1), which is crucial for solving the general BCLS (in Section 2.2) that we refer to as the optimization version of the problem.

For each sensor $s_{i} \in S$, we call the right (resp., left) endpoint of the covering interval of $s_{i}$ the right (resp., left) extension of $s_{i}$. Each of the right and left extensions of $s_{i}$ is an extension of $s_{i}$. Denote by $p\left(x^{\prime}\right)$ the point on the $x$-axis whose coordinate is $x^{\prime}$, and denote by $p^{+}\left(x^{\prime}\right)$ (resp., $\left.p^{-}\left(x^{\prime}\right)\right)$ a point to the right (resp., left) of $p\left(x^{\prime}\right)$ and infinitely close to $p\left(x^{\prime}\right)$. The concept of $p^{+}\left(x^{\prime}\right)$ and $p^{-}\left(x^{\prime}\right)$ is only used to explain the algorithms, and we never need to find such a point explicitly in the algorithm. Let $\lambda^{*}$ denote the maximum sensor moving distance in an optimal solution for the optimization version of the general BCLS problem. Note that we can easily determine whether $\lambda^{*}=0$, say, in $O(n \log n)$ time. Henceforth, we assume $\lambda^{*}>0$.

\subsection{The Decision Version of the General BCLS}

Given any value $\lambda$, the decision version is to determine whether there is a feasible solution in which the maximum sensor movement is at most $\lambda$. Clearly, there is a feasible solution if and only if $\lambda \geq \lambda^{*}$. We show that after $O(n \log n)$ time preprocessing, for any $\lambda$, we can determine whether $\lambda \geq \lambda^{*}$ in $O(n)$ time. We explore some properties of a feasible solution in Section 2.1.1, describe our decision algorithm in Section 2.1.2, argue its correctness in Section 2.1.3, and discuss 
its implementation in Section 2.1.4. In Section 2.1.5, we show that by extending the algorithm, we can also determine whether $\lambda>\lambda^{*}$ in the same time bound; this is particularly useful to our optimization algorithm in Section 2.2 .

\subsubsection{Preliminaries}

By a sensor configuration, we refer to a specification of where each sensor $s_{i} \in S$ is located. By this definition, the input is a configuration in which each sensor $s_{i}$ is located at $x_{i}$. The displacement of a sensor in a configuration $C$ is the distance between the position of the sensor in $C$ and its original position in the input. A configuration $C$ is a feasible solution for the distance $\lambda$ if the sensors in $C$ form a barrier coverage of $B$ (i.e., the union of the covering intervals of the sensors in $C$ contains $B$ ) and the displacement of each sensor is at most $\lambda$. In a feasible solution, a subset $S^{\prime} \subseteq S$ is called a solution set if the sensors in $S^{\prime}$ form a barrier coverage; of course, $S$ itself is also a solution set. A feasible solution may have multiple solution sets. A sensor $s_{i}$ in a solution set $S^{\prime}$ is said to be critical with respect to $S^{\prime}$ if $s_{i}$ covers a point on $B$ that is not covered by any other sensor in $S^{\prime}$. If every sensor in $S^{\prime}$ is critical, then $S^{\prime}$ is called a critical set.

Given any value $\lambda$, if $\lambda \geq \lambda^{*}$, our decision algorithm will find a critical set and determine the order in which the sensors of the critical set will appear in a feasible solution for $\lambda$. For the purpose of giving some intuition and later showing the correctness of our algorithm, we first explore some properties of a critical set.

Consider a critical set $S^{c}$. For each sensor $s \in S^{c}$, we call the set of points on $B$ that are covered by $s$ but not covered by any other sensor in $S^{c}$ the exclusive coverage of $s$.

Observation 1 The exclusive coverage of each sensor in a critical set $S^{c}$ is a continuous portion of the barrier $B$.

Proof: Assume to the contrary the exclusive coverage of a sensor $s \in S^{c}$ is not a continuous portion of $B$. Then there must be at least one sensor $s^{\prime} \in S^{c}$ whose covering interval is between two consecutive continuous portions of the exclusive coverage of $s$. But that would mean $s^{\prime}$ is not critical since the covering interval of $s^{\prime}$ is contained in that of $s$. Hence, the observation holds.

For a critical set $S^{c}$ in a feasible solution $S O L$, we define the cover order of the sensors in $S^{c}$ as the order of these sensors in $S O L$ such that their exclusive coverages are from left to right.

Observation 2 The cover order of the sensors of a critical set $S^{c}$ in a feasible solution $S O L$ is consistent with the left-to-right order of the positions of these sensors in SOL. Further, the cover order is also consistent with the order of the right (resp., left) extensions of these sensors in SOL.

Proof: Consider any two sensors $s_{i}$ and $s_{j}$ in $S^{c}$ with ranges $r_{i}$ and $r_{j}$, respectively. Without loss of generality, assume $s_{i}$ is to the left of $s_{j}$ in the cover order, i.e., the exclusive coverage of $s_{i}$ is to the left of that of $s_{j}$ in $S O L$. Let $y_{i}$ and $y_{j}$ be the positions of $s_{i}$ and $s_{j}$ in $S O L$, respectively. To prove the observation, it suffices to show $y_{i}<y_{j}, y_{i}+r_{i}<y_{j}+r_{j}$, and $y_{i}-r_{i}<y_{j}-r_{j}$.

Let $p$ be a point in the exclusive coverage of $s_{j}$. We also use $p$ to denote its coordinate on the $x$-axis. Then $p$ is not covered by $s_{i}$, implying either $p>y_{i}+r_{i}$ or $p<y_{i}-r_{i}$. But, the latter case cannot hold (otherwise, the exclusive coverage of $s_{i}$ would be to the right of that of $s_{j}$ ). Since $p$ is covered by $s_{j}$, we have $p \leq y_{j}+r_{j}$. Therefore, $y_{i}+r_{i}<p \leq y_{j}+r_{j}$. By using a symmetric argument, we can also prove $y_{i}-r_{i}<y_{j}-r_{j}$ (we omit the details). Clearly, the two inequalities $y_{i}+r_{i}<y_{j}+r_{j}$ and $y_{i}-r_{i}<y_{j}-r_{j}$ imply $y_{i}<y_{j}$. The observation thus holds. 
An interval $I$ of $B$ is called a left-aligned interval if the left endpoint of $I$ is at 0 (i.e., $I$ is of the form $\left[0, x^{\prime}\right]$ or $\left.\left[0, x^{\prime}\right)\right)$. A set of sensors is said to be in attached positions if the union of their covering intervals is a continuous interval of the $x$-axis whose length is equal to the sum of the lengths of these covering intervals. Two intervals of the $x$-axis overlap if they intersect each other (even at only one point).

\subsubsection{The Algorithm Description}

Initially, we move all sensors of $S$ to the right by the distance $\lambda$, i.e., for each $1 \leq i \leq n$, we move $s_{i}$ to the position $x_{i}^{\prime}=x_{i}+\lambda$. Let $C_{0}$ denote the resulting configuration. Clearly, there is a feasible solution for $\lambda$ if and only if we can move the sensors in $C_{0}$ to the left by at most $2 \lambda$ to form a coverage of $B$. Thus, henceforth we only need to consider moving the sensors to the left. Recall that we have assumed that the extensions of any two distinct sensors are different; hence in $C_{0}$, the extensions of all sensors are also different.

Our algorithm takes a greedy approach. It seeks to find sensors to cover $B$ from left to right, in at most $n$ steps. If $\lambda \geq \lambda^{*}$, the algorithm will end up with a critical set $S^{c}$ of sensors along with the destinations for all these sensors. In theory, the other sensors in $S \backslash S^{c}$ can be anywhere such that their displacements are at most $\lambda$; but in the solution found by our algorithm, they are at the same positions as in $C_{0}$. If a sensor is at the same position as in $C_{0}$, we say it stands still.

In step $i$ (initially, $i=1$ ), using the configuration $C_{i-1}$ produced in step $i-1$ and based on certain criteria, we find a sensor $s_{g(i)}$ and determine its destination $y_{g(i)}$, where $g(i)$ is the index of the sensor in $S$ and $y_{g(i)} \in\left[x_{g(i)}^{\prime}-2 \lambda, x_{g(i)}^{\prime}\right]$. We then move the sensor $s_{g(i)}$ to $y_{g(i)}$ to obtain a new configuration $C_{i}$ from $C_{i-1}$ (if $y_{g(i)}=x_{g(i)}^{\prime}$, then we need not move $s_{g(i)}$, and $C_{i}$ is the same as $\left.C_{i-1}\right)$. Let $R_{i}=y_{g(i)}+r_{g(i)}$ (i.e., the right extension of $s_{g(i)}$ in $C_{i}$ ). Assume $R_{0}=0$. Let $S_{i}=S_{i-1} \cup\left\{s_{g(i)}\right\}\left(S_{0}=\emptyset\right.$ initially). We will show that the sensors in $S_{i}$ together cover the left-aligned interval [0, $R_{i}$ ]. If $R_{i} \geq L$, we have found a feasible solution with a critical set $S^{c}=S_{i}$, and terminate the algorithm. Otherwise, we proceed to step $i+1$. Further, it is possible that a desired sensor $s_{g(i)}$ cannot be found, in which case we terminate the algorithm and report $\lambda<\lambda^{*}$. Below we give the details, and in particular, discuss how to determine the sensor $s_{g(i)}$ in each step.

Before discussing the first step, we provide some intuition. Let $S_{l}$ consist of the sensors whose right extensions are at most 0 in $C_{0}$. We claim that since $L>0$, no sensor in $S_{l}$ can be in a critical set of a feasible solution if $\lambda^{*} \leq \lambda$. Indeed, because all sensors have been moved to their rightmost possible positions in $C_{0}$, if no sensor in $S_{l}$ has a right extension at 0 in $C_{0}$, then the claim trivially holds; otherwise, suppose $s_{t}$ is such a sensor. Assume to the contrary that $s_{t}$ is in a critical set $S^{c}$. Then $p(0)$ is the only point on $B$ that can be covered by $s_{t}$. Since $L>0$, there must be another sensor in $S^{c}$ that also covers $p(0)$ (otherwise, no sensor in $S^{c}$ would cover the point $p^{+}(0)$ ). Hence, $s_{t}$ is not critical with respect to $S^{c}$, a contradiction. The claim thus follows. Therefore, we need not consider the sensors in $S_{l}$ since they do not help in forming a feasible solution.

In step 1, we determine the sensor $s_{g(1)}$, as follows. Define $S_{11}=\left\{s_{j} \mid x_{j}^{\prime}-r_{j} \leq 0<x_{j}^{\prime}+r_{j}\right\}$ (see Fig. 1), i.e., $S_{11}$ consists of all sensors covering the point $p(0)$ in $C_{0}$ except any sensor whose right extension is 0 (but if the left extension of a sensor is 0 , the sensor is included in $S_{11}$ ). In other words, $S_{11}$ consists of all sensors covering the point $p^{+}(0)$ in $C_{0}$. If $S_{11} \neq \emptyset$, then we choose the sensor in $S_{11}$ whose right extension is the largest as $s_{g(1)}$ (e.g., $s_{i}$ in Fig. 11), and let $y_{g(1)}=x_{g(1)}^{\prime}$. Note that since the extensions of all sensors in $C_{0}$ are different, the sensor $s_{g(1)}$ is unique. If $S_{11}=\emptyset$, then define $S_{12}$ as the set of sensors whose left extensions are larger than 0 and at most $2 \lambda$ (e.g., see Fig. (2). If $S_{12}=\emptyset$, then we terminate the algorithm and report $\lambda<\lambda^{*}$. Otherwise, we choose the sensor in $S_{12}$ whose right extension is the smallest as $s_{g(1)}$ (e.g., $s_{i}$ in Fig. 2), and let $y_{g(1)}=r_{g(1)}$ 


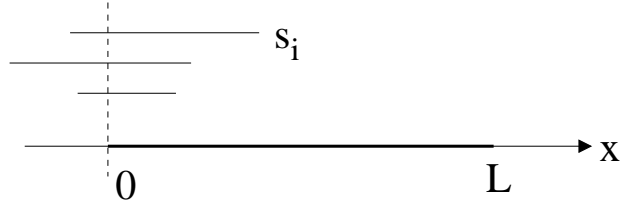

Figure 1: The set $S_{11}$ consists of the three sensors whose covering intervals are shown, and $s_{g(1)}$ is $s_{i}$.

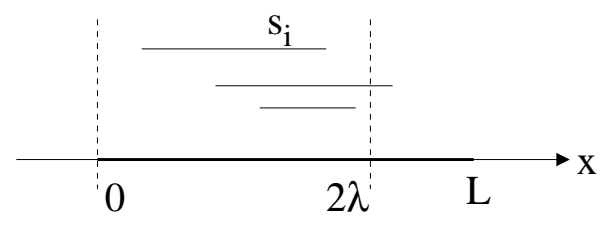

Figure 2: The set $S_{12}$ consists of the three sensors whose covering intervals are shown, and $s_{g(1)}$ is $s_{i}$ if $S_{11}=\emptyset$.

(i.e., the left extension of $s_{g(1)}$ is at 0 after it is moved to the destination $y_{g(1)}$ ).

If the algorithm is not terminated, then we move $s_{g(1)}$ to $y_{g(1)}$, yielding a new configuration $C_{1}$. Let $S_{1}=\left\{s_{g(1)}\right\}$, and $R_{1}$ be the right extension of $s_{g(1)}$ in $C_{1}$. If $R_{1} \geq L$, we have found a feasible solution $C_{1}$ with the critical set $S_{1}$, and terminate the algorithm. Otherwise, we proceed to step 2.

The general step is very similar to step 1 . Consider step $i$ for $i>1$. We determine the sensor $s_{g(i)}$, as follows. Let $S_{i 1}$ be the set of sensors covering the point $p^{+}\left(R_{i-1}\right)$ in the configuration $C_{i-1}$. If $S_{i 1} \neq \emptyset$, we choose the sensor in $S_{i 1}$ with the largest right extension as $s_{g(i)}$ and let $y_{g(i)}=x_{g(i)}^{\prime}$. Otherwise, let $S_{i 2}$ be the set of sensors whose left extensions are larger than $R_{i-1}$ and at most $R_{i-1}+2 \lambda$. If $S_{i 2}=\emptyset$, we terminate the algorithm and report $\lambda<\lambda^{*}$. Otherwise, we choose the sensor in $S_{i 2}$ with the smallest right extension as $s_{g(i)}$ and let $y_{g(i)}=R_{i-1}+r_{g(i)}$. If the algorithm is not terminated, we move $s_{g(i)}$ to $y_{g(i)}$ and obtain a new configuration $C_{i}$. Let $S_{i}=S_{i-1} \cup\left\{s_{g(i)}\right\}$. Let $R_{i}$ be the right extension of $s_{g(i)}$ in $C_{i}$. If $R_{i} \geq L$, we have found a feasible solution $C_{i}$ with the critical set $S_{i}$ and terminate the algorithm. Otherwise, we proceed to step $i+1$. If the sensor $s_{g(i)}$ is from $S_{i 1}$ (resp., $S_{i 2}$ ), then we call it the type $I$ (resp., type $I I$ ) sensor.

Since there are $n$ sensors in $S$, the algorithm is terminated in at most $n$ steps. This finishes the description of our algorithm.

\subsubsection{The Correctness of the Algorithm}

Based on the description of our algorithm, we have the following lemma.

Lemma 1 At the end of step $i$, suppose the algorithm produces the set $S_{i}$ and the configuration $C_{i}$; then $S_{i}$ and $C_{i}$ have the following properties.

(a) $S_{i}$ consists of sensors that are type I or type II.

(b) For each sensor $s_{g(j)} \in S_{i}$ with $1 \leq j \leq i$, if $s_{g(j)}$ is of type $I$, then it stands still (i.e., its position in $C_{i}$ is the same as that in $\left.C_{0}\right)$; otherwise, its left extension is at $R_{j-1}$, and $s_{g(j)}$ and $s_{g(j-1)}$ are in attached positions if $j>1$.

(c) The interval on $B$ covered by the sensors in $S_{i}$ is $\left[0, R_{i}\right]$.

(d) For each $1<j \leq i$, the right extension of $s_{g(j)}$ is larger than that of $s_{g(j-1)}$.

(e) For each $1 \leq j \leq i, s_{g(j)}$ is the only sensor in $S_{i}$ that covers the point $p^{+}\left(R_{j-1}\right)$ (with $\left.R_{0}=0\right)$.

Proof: The first three properties are trivially true according to the algorithm description.

For property (d), note that the right extension of $s_{g(j)}$ (resp., $\left.s_{g(j-1)}\right)$ is $R_{j}$ (resp., $R_{j-1}$ ). According to our algorithm, the sensor $s_{g(j)}$ covers the point $p^{+}\left(R_{j-1}\right)$, implying that $R_{j}>R_{j-1}$. Hence, property (d) holds.

For property (e), note that the sensor $s_{g(j)}$ (which is determined in step $j$ ) always covers $p^{+}\left(R_{j-1}\right)$. Consider any other sensor $s_{g(t)} \in S_{i}$. If $t<j$, then the right extension of $s_{g(t)}$ is at most 
$R_{j-1}$, and thus $s_{g(t)}$ cannot cover $p^{+}\left(R_{j-1}\right)$. If $t>j$, then depending on whether $s_{g(t)} \in S_{t 1}$ or $s_{g(t)} \in S_{t 2}$, there are two cases. If $s_{g(t)} \in S_{t 2}$, then the left extension of $s_{g(t)}$ is $R_{t-1}$, which is larger than $R_{j-1}$, and thus $s_{g(t)}$ cannot cover $p^{+}\left(R_{j-1}\right)$ in $C_{i}$. Otherwise (i.e., $\left.s_{g(t)} \in S_{t 1}\right), s_{g(t)}$ stands still. Assume to the contrary that $s_{g(t)}$ covers $p^{+}\left(R_{j-1}\right)$ in $C_{i}$. Then $s_{g(t)}$ must have been in $S_{j 1}$ in step $j$ within the configuration $C_{j-1}$. This implies $S_{j 1} \neq \emptyset, s_{g(j)} \in S_{j 1}$, and $s_{g(j)}$ stands still. Since $R_{t}$ is the right extension of $s_{g(t)}$ and $R_{j}$ is the right extension of $s_{g(j)}$, by property (d), for $t>j$, we have $R_{t}>R_{j}$. Since $R_{t}>R_{j}$ (i.e., the right extension of $s_{g(j)}$ is smaller than that of $s_{g(t)}$ ), the algorithm cannot choose $s_{g(j)}$ from $S_{j 1}$ in step $j$, which is a contradiction. Therefore, $s_{g(t)}$ cannot cover the point $p^{+}\left(R_{j-1}\right)$. Property (e) thus holds.

At its termination, our algorithm either reports $\lambda \geq \lambda^{*}$ or $\lambda<\lambda^{*}$. To argue the correctness of the algorithm, below we will show that if the algorithm reports $\lambda \geq \lambda^{*}$, then indeed there is a feasible solution for $\lambda$ and our algorithm finds one; otherwise, there is no feasible solution for $\lambda$.

Suppose in step $i$, our algorithm reports $\lambda \geq \lambda^{*}$. Then according to the algorithm, it must be $R_{i} \geq L$. By Lemma 1(c) and 1(e), $C_{i}$ is a feasible solution and $S_{i}$ is a critical set. Further, by Lemma 1(d) and Observation 2, the cover order of the sensors in $S_{i}$ is $s_{g(1)}, s_{g(2)}, \ldots, s_{g(i)}$.

Next, we show that if the algorithm reports $\lambda<\lambda^{*}$, then certainly there is no feasible solution for $\lambda$. This is almost an immediate consequence of the following lemma.

Lemma 2 Suppose $S_{i}^{\prime}$ is the set of sensors in the configuration $C_{i}$ whose right extensions are at most $R_{i}$. Then the interval $\left[0, R_{i}\right]$ is the largest possible left-aligned interval that can be covered by the sensors of $S_{i}^{\prime}$ such that the displacement of each sensor in $S_{i}^{\prime}$ is at most $\lambda$.

Proof: In this proof, when we say an interval is covered by the sensors of $S_{i}^{\prime}$, we mean (without explicitly stating) that the displacement of each sensor in $S_{i}^{\prime}$ is at most $\lambda$.

We first prove a key claim: If $C$ is a configuration for the sensors of $S_{i}^{\prime}$ such that a left-aligned interval $\left[0, x^{\prime}\right]$ is covered by the sensors of $S_{i}^{\prime}$, then there always exists a configuration $C^{*}$ for $S_{i}^{\prime}$ in which the interval $\left[0, x^{\prime}\right]$ is still covered by the sensors of $S_{i}^{\prime}$ and for each $1 \leq j \leq i$, the position of the sensor $s_{g(j)}$ in $C^{*}$ is $y_{g(j)}$, where $g(j)$ and $y_{g(j)}$ are the values computed by our algorithm.

As similar to our discussion in Section 2.1.1, the configuration $C$ for $S_{i}^{\prime}$ always has a critical set for covering the interval $\left[0, x^{\prime}\right]$. Let $S_{C}$ be such a critical set of $C$.

We prove the claim by induction. We first show the base case: Suppose there is a configuration $C$ for the sensors of $S_{i}^{\prime}$ in which a left-aligned interval $\left[0, x^{\prime}\right]$ is covered by the sensors of $S_{i}^{\prime}$; then there is a configuration $C_{1}^{\prime}$ for the sensors of $S_{i}^{\prime}$ in which the interval $\left[0, x^{\prime}\right]$ is still covered by the sensors of $S_{i}^{\prime}$ and the position of the sensor $s_{g(1)}$ in $C_{1}^{\prime}$ is $y_{g(1)}$.

Let $t=g(1)$. If the position of $s_{t}$ in $C$ is $y_{t}$, then we are done (with $C_{1}^{\prime}=C$ ). Otherwise, let $y_{t}^{\prime}$ be the position of $s_{t}$ in $C$, with $y_{t}^{\prime} \neq y_{t}$. Depending on $s_{t} \in S_{11}$ or $s_{t} \in S_{12}$, there are two cases.

If $s_{t} \in S_{11}$, then $y_{t}=x_{t}^{\prime}$. Since $y_{t}$ is the rightmost position to which the sensor $s_{t}$ is allowed to move and $y_{t}^{\prime} \neq y_{t}$, we have $y_{t}^{\prime}<y_{t}$. Depending on whether $s_{t}$ is in the critical set $S_{C}$, there further are two subcases.

If $s_{t} \notin S_{C}$, then by the definition of a critical set, the sensors in $S_{C}$ form a coverage of $\left[0, x^{\prime}\right]$ regardless of where $s_{t}$ is. If we move $s_{t}$ to $y_{t}$ (and other sensors keep the same positions as in $C$ ) to obtain a new configuration $C_{1}^{\prime}$, then the sensors of $S_{i}^{\prime}$ still form a coverage of $\left[0, x^{\prime}\right]$.

If $s_{t} \in S_{C}$, then because $y_{t}>y_{t}^{\prime}$, if we move $s_{t}$ from $y_{t}^{\prime}$ to $y_{t}, s_{t}$ is moved to the right. Since $s_{t} \in S_{11}$, when $s_{t}$ is at $y_{t}, s_{t}$ still covers the point $p(0)$. Thus, moving $s_{t}$ from $y_{t}^{\prime}$ to $y_{t}$ does not cause $s_{t}$ to cover a smaller sub-interval of $\left[0, x^{\prime}\right]$. Hence, by moving $s_{t}$ to $y_{t}$, we obtain a new configuration $C_{1}^{\prime}$ in which the sensors of $S_{i}^{\prime}$ still form a coverage of $\left[0, x^{\prime}\right]$.

This completes the proof for the case $s_{t} \in S_{11}$. 

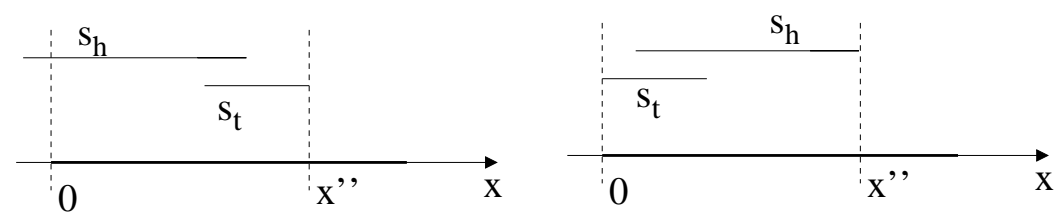

Figure 3: Illustrating the switch operation on $s_{t}$ and $s_{h}$ : The left figure is before the switch and the right one is after the switch.

If $s_{t} \in S_{12}$, then according to our algorithm, $S_{11}=\emptyset$ in this case, and $s_{t}$ is the sensor in $S_{12}$ with the smallest right extension in $C_{0}$. If $s_{t} \notin S_{C}$, then by the same argument as above, we can obtain a configuration $C_{1}^{\prime}$ in which the interval $\left[0, x^{\prime}\right]$ is still covered by the sensors of $S_{i}^{\prime}$ and the position of the sensor $s_{t}$ in $C_{1}^{\prime}$ is $y_{t}$. Below, we discuss the case when $s_{t} \in S_{C}$.

In $S_{C}$, some sensors must cover the point $p(0)$ in $C$. Let $S^{\prime}$ be the set of sensors in $S_{C}$ that cover $p(0)$ in $C$. If $s_{t} \in S^{\prime}$, then it is easy to see that $y_{t}^{\prime}<y_{t}$ since $y_{t}$ is the rightmost position for $s_{t}$ to cover $p(0)$. In this case, again, by the same argument as above, we can always move $s_{t}$ to the right from $y_{t}^{\prime}$ to $y_{t}$ to obtain a configuration $C_{1}^{\prime}$ in which the interval $\left[0, x^{\prime}\right]$ is still covered by the sensors of $S_{i}^{\prime}$. Otherwise (i.e., $s_{t} \notin S^{\prime}$ ), we show below that we can always move $s_{t}$ to $y_{t}$ by switching the relative positions of $s_{t}$ and some other sensors in $S_{C}$.

An easy observation is that each sensor in $S^{\prime}$ must be in $S_{12}$. Consider an arbitrary sensor $s_{h} \in S^{\prime}$. Since $s_{t}$ is the sensor in $S_{12}$ with the smallest right extension in $C_{0}$, the right extension of $s_{h}$ is larger than that of $s_{t}$ in $C_{0}$. Depending on whether the covering intervals of $s_{t}$ and $s_{h}$ overlap in $C$, there are two subcases.

If the covering intervals of $s_{t}$ and $s_{h}$ overlap in $C$, then let $\left[0, x^{\prime \prime}\right]$ be the left-aligned interval that is covered by $s_{t}$ and $s_{h}$ in $C$ (see Fig. 3). If we switch their relative positions by moving $s_{t}$ to $y_{t}$ and moving $y_{h}$ to $x^{\prime \prime}-r_{h}$ (i.e., the left extension of $s_{t}$ is at 0 and the right extension of $s_{h}$ is at $x^{\prime \prime}$ ), then these two sensors still cover $\left[0, x^{\prime \prime}\right]$ (see Fig. 3), and thus the sensors in $S_{i}^{\prime}$ still form a coverage of $\left[0, x^{\prime \prime}\right]$. Further, after the above switch operation, the displacements of these two sensors are no bigger than $\lambda$. To see this, clearly, the displacement of $s_{t}$ is at most $\lambda$. For the sensor $s_{h}$, it is easy to see that the switch operation moves $s_{h}$ to the right. Since $s_{t}$ covers $p\left(x^{\prime \prime}\right)$ in $C, x^{\prime \prime}$ is no larger than the right extension of $s_{t}$ in $C_{0}$, which is smaller than that of $s_{h}$ in $C_{0}$ since $s_{h}$ is in $S_{12}$. Thus, $x^{\prime \prime}$ is smaller than $x_{h}^{\prime}+r_{h}$, implying that the position of $s_{h}$ after the switch operation is still to the left of its position in $C_{0}$. Hence, after the switch operation, the displacement of $s_{h}$ is no bigger than $\lambda$. In summary, after the switch operation, we obtain a new configuration $C_{1}^{\prime}$ in which the interval $\left[0, x^{\prime}\right]$ is still covered by the sensors of $S_{i}^{\prime}$ and the position of the sensor $s_{t}$ in $C_{1}^{\prime}$ is $y_{t}$.

If the covering intervals of $s_{t}$ and $s_{h}$ do not overlap in $C$, then suppose the sensors in the critical set $S_{C}$ between $s_{h}$ and $s_{t}$ are $s_{h}=s_{f(0)}, s_{f(1)}, s_{f(2)}, \ldots, s_{f(m)}, s_{t}$, in the cover order. Clearly, the covering intervals of any two consecutive sensors in this list overlap in $C$. In the following, we perform a sequence of "switch operations" to obtain a configuration $C_{1}^{\prime}$ in which $\left[0, x^{\prime}\right]$ is covered by the sensors of $S_{i}^{\prime}$ and the location of the sensor $s_{t}$ is $y_{t}$. Let $Q=\left\{s_{f(0)}, s_{f(1)}, s_{f(2)}, \ldots, s_{f(m)}\right\}$.

Indeed, this is the most difficult case*.

For any sensor $s_{k}$ of $S$ and any configuration $C^{\prime}$, we use $x_{k}\left(C^{\prime}\right)$ to denote its location in $C^{\prime}$. Recall that $x_{k}^{\prime}$ is the location of $s_{k}$ in $C_{0}$. We use $I\left(s_{k}\right)$ to denote the covering interval of $s_{k}$. We say that $s_{k}$ is valid in a configuration $C^{\prime}$ if the displacement of $s_{k}$ in $C^{\prime}$ is at most $\lambda$ (i.e., $\left.x_{k}\left(C^{\prime}\right) \in\left[x_{k}^{\prime}-2 \lambda, x_{k}^{\prime}\right]\right)$. We say that a configuration $C^{\prime}$ is valid if every sensor of $S$ is valid in $C^{\prime}$.

* The proof for this case in the previous version of this paper [4] is based on the assumption that every sensor of $Q$ is in $S_{12}$. However, the assumption is not true because it is possible that some sensors of $Q$ are not in $S_{12}$. Therefore, the previous proof is not correct. Here, we provide a new proof for this case. 


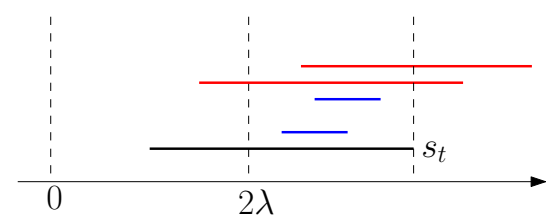

Figure 4: Illustrating the special and normal sensors of $Q$ when they are in $C_{0}$ : each segment represents the covering interval of a sensor of $Q$ in $C_{0}$; the black segment is for $s_{t}$; the red segments are for normal sensors and the blue segments are for special sensors.

Consider any sensor $s_{f(j)}$ in $Q$. If the right extension of $s_{f(j)}$ is strictly less than the right extension of $s_{t}$ in $C_{0}$, then we say $s_{f(j)}$ is a special sensor; otherwise it is a normal sensor (see Fig 4). Note that all sensors of $S_{12}$ are normal sensors but not every normal sensor is in $S_{12}$. If a normal sensor is not in $S_{12}$, then its left extension must be larger than $2 \lambda$ in $C_{0}$.

We begin with considering the last sensor $s_{f(m)}$ of the sequence $Q$. Depending on whether $s_{f(m)}$ is a normal sensor or a special sensor, there are two cases.

The normal sensor case. If $s_{f(m)}$ is a normal sensor, then let $x_{1}^{\prime \prime}$ be the maximum of 0 and the

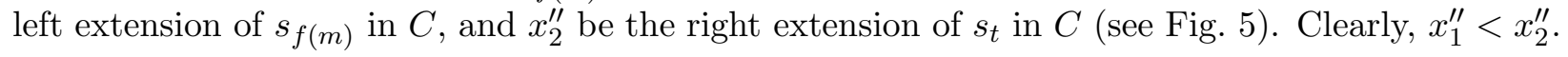
We perform a switch operation on $s_{t}$ and $s_{f(m)}$ by moving $s_{t}$ to the left and moving $s_{f(m)}$ to the

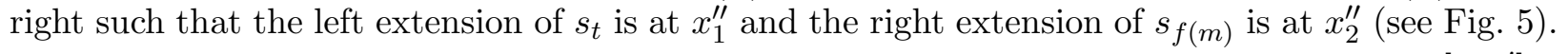
It is easy to see that after this switch operation, the sensors in $S_{C}$ still form a coverage of $\left[0, x^{\prime}\right]$.

Let $C^{\prime}$ be the new configuration after the above switch operation. Next we show that $C^{\prime}$ is still valid. To this end, it is sufficient to show both $s_{f(m)}$ and $s_{t}$ are valid in $C^{\prime}$.
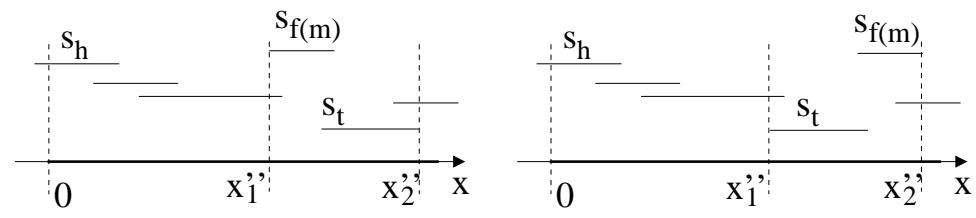

Figure 5: Illustrating the switch between $s_{t}$ and $s_{f(m)}$.

We first consider $s_{t}$. Our goal is to show that $x_{t}\left(C^{\prime}\right) \in\left[x_{t}^{\prime}-2 \lambda, x_{t}^{\prime}\right]$. On the one hand, since the left extension of $s_{t}$ in $C^{\prime}$ is at $x_{1}^{\prime \prime} \geq 0$, it holds that $x_{t}\left(C^{\prime}\right) \geq y_{t} \geq x_{t}^{\prime}-2 \lambda$. On the other hand, since $s_{t}$ is valid in $C$ and $s_{t}$ has been moved leftwards from $x_{t}(C)$ to $x_{t}\left(C^{\prime}\right)$, we obtain that $x_{t}\left(C^{\prime}\right) \leq x_{t}(C) \leq x_{t}^{\prime}$. This proves that $s_{t}$ is valid in $C^{\prime}$.

We then consider $s_{f(m)}$. Let $k=f(m)$. Our goal is to show that $x_{k}\left(C^{\prime}\right) \in\left[x_{k}^{\prime}-2 \lambda, x_{k}^{\prime}\right]$. On the one hand, since $s_{k}$ is valid in $C$ and $s_{k}$ has been moved rightwards from $x_{k}(C)$ to $x_{k}\left(C^{\prime}\right)$, we obtain that $x_{k}^{\prime}-2 \lambda \leq x_{k}(C) \leq x_{k}\left(C^{\prime}\right)$. On the other hand, since $s_{k}$ is a normal sensor, the right extension of $s_{k}$ is larger than that of $s_{t}$ in $C_{0}$. However, the right extension of $s_{k}$ in $C^{\prime}$ is at $x_{2}^{\prime \prime}$, which is the right extension of $s_{t}$ in $C$. This implies that comparing with its location $x_{k}^{\prime}$ in $C_{0}, s_{k}$ must have been moved leftwards to reach $x_{k}\left(C^{\prime}\right)$. In other words, $x_{k}\left(C^{\prime}\right) \leq x_{k}^{\prime}$. This proves that $x_{k}\left(C^{\prime}\right) \in\left[x_{k}^{\prime}-2 \lambda, x_{k}^{\prime}\right]$, and thus, $s_{k}$ is valid in $C^{\prime}$.

Thus, $C^{\prime}$ is a valid configuration in which $\left[0, x^{\prime}\right]$ is still covered by the sensors of $S_{C}$. But the sensors between $s_{h}$ and $s_{t}$ in $C^{\prime}$ are $s_{f(1)}, s_{f(2)}, \ldots, s_{f(m-1)}$, which has one sensor less than before. Let $Q=\left\{s_{f(0)}, s_{f(1)}, s_{f(2)}, \ldots, s_{f(m-1)}\right\}$. We proceed to apply the same argument on $Q$ and $C^{\prime}$.

The special sensor case. If $s_{f(m)}$ is a special sensor, this case is more complicated. Let $s_{f(j)}$ be the last normal sensor in the sequence $Q$ for some $j$ with $0 \leq j \leq m-1$. Note that such a 
normal sensor must exist in $Q$ because $s_{f(0)}=s_{h}$ is a normal sensor. Let $\mathcal{I}=[\alpha, \beta]$ be the union of the covering intervals of $s_{f(j+1)}, s_{f(j+2)}, \ldots, s_{f(m)}$ in $C$. Let $\mathcal{I}^{\prime}=\left[\alpha^{\prime}, \beta^{\prime}\right]$ be the union of $\mathcal{I}$ and the covering intervals of $s_{f(j)}$ and $s_{t}$ in $C$. For ease of exposition, we assume $\mathcal{I}^{\prime} \subseteq\left[0, x^{\prime}\right]$ (since otherwise we could apply similar argument on the interval $[0, \infty) \cap I^{\prime}$, as we did for the above normal sensor case). Note that since all sensors of $Q \cup\left\{s_{f(j)}, s_{t}\right\}$ are in the critical set $S_{C}$ and their order follows the cover order, the left extension of $s_{f(j)}$ is at $\alpha^{\prime}$ and the right extension of $s_{t}$ is at $\beta^{\prime}$ in $C$.

Consider any sensor $s_{f(k)}$ with $j+1 \leq k \leq m$. Since $s_{f(k)}$ is a special sensor, we claim that the left extension of $s_{f(k)}$ in $C_{0}$ is larger than $2 \lambda$ (see Fig. 4). Indeed, assume to the contrary that this is not true. Then, since $S_{11}=\emptyset$ and $s_{f(k)} \notin S_{12}$, the right extension of $s_{f(k)}$ in $C_{0}$ must be at most 0 . However, this implies that $s_{f(k)}$ cannot be in the critical set $S_{C}$ for covering the interval $\left[0, x^{\prime}\right]$, incurring contradiction. This proves the claim.

Let $Q^{\prime}=\left\{s_{f(j+1)}, s_{f(j+2)}, \ldots, s_{f(m)}\right\}$. Define $Q_{1}^{\prime}$ to be the set of sensors of $Q^{\prime}$ whose right extensions are less than the left extension of $s_{f(j)}$ in $C_{0}$. Let $Q_{2}^{\prime}=Q^{\prime} \backslash Q_{1}^{\prime}$ (see Fig. 6).

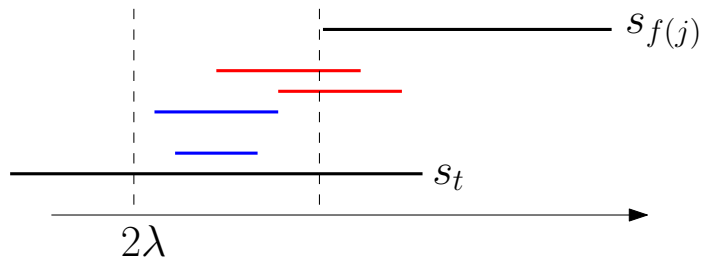

Figure 6: Illustrating $Q_{1}^{\prime}$ and $Q_{2}^{\prime}$. The segments are covering intervals of sensors in $Q^{\prime} \cup\left\{s_{t}, s_{f(j)}\right\}$ in $C_{0}$. The set $Q_{1}^{\prime}$ (resp., $Q_{2}^{\prime}$ ) consists of the two sensors whose covering intervals are blue (resp., red).

Note that if $s_{f(j)}$ is in $S_{k 2}$, then $Q_{1}^{\prime}=\emptyset$. To see this, suppose $s_{f(j)}$ is in $S_{k 2}$. Then, the left extension of $s_{f(j)}$ in $C_{0}$ is at most $2 \lambda$. Since each sensor of $Q^{\prime}$ is a special sensor, its right extension must be larger than $2 \lambda$. Thus, every sensor of $Q^{\prime}$ has its right extension larger than the left extension of $s_{f(j)}$ in $C_{0}$. Hence, $Q_{1}^{\prime}=\emptyset$.

Let $Q_{1}^{\prime}=\left\{s_{f_{1}(1)}, s_{f_{1}(2)}, \ldots, s_{f_{1}\left(m_{1}\right)}\right\}$ sorted by their index order in $Q^{\prime}$.

In the sequel, we will perform a sequence of operations to move sensors of $Q^{\prime} \cup\left\{s_{f(j)}, s_{t}\right\}$ in $C$ to obtain a new configuration $C^{\prime}$. Roughly speaking, in $C^{\prime}$, the order of the sensors of $Q^{\prime}$ from left to right are sensors of $Q_{1}^{\prime}, s_{t}$, sensors of $Q_{2}^{\prime}$, and $s_{f(j)}$. We will show that after the operations, $\mathcal{I}^{\prime}$ is still covered by the sensors of $Q^{\prime} \cup\left\{s_{f(j)}, s_{t}\right\}$ and $C^{\prime}$ is a valid configuration. In the following discussion, for each sensor $s_{k} \in Q^{\prime}, x_{k}\left(C^{\prime}\right)$ refers to its location in $C^{\prime}$ that we are going to set.

1. We first consider sensors of $Q_{1}^{\prime}$.

We consider the sensors of $Q_{1}^{\prime}$ by their index order. By the definition of $\mathcal{I}^{\prime}=\left[\alpha^{\prime}, \beta^{\prime}\right]$, the left extension of $s_{f_{1}(1)}$ in $C$ is larger than $\alpha^{\prime}$. We move $s_{f_{1}(1)}$ leftwards until its left extension is at $\alpha^{\prime}$.

Then, we consider $s_{f_{1}(2)}$. Clearly, either $I\left(s_{f_{1}(2)}\right)$ intersects $I\left(s_{f_{1}(1)}\right)$ or $I\left(s_{f_{1}(2)}\right)$ is to the right of $I\left(s_{f_{1}(1)}\right)$. If the former case happens, we do not move $s_{f_{1}(2)}$; otherwise, we move $s_{f_{1}(2)}$ leftwards until its left extension is at the right extension of $s_{f_{1}(1)}$.

We continue to consider other sensors of $Q_{1}^{\prime}$. In general, suppose sensor $s_{f_{1}(k)}$ has been considered as above. Then, we consider sensor $s_{f_{1}(k+1)}$. Either $I\left(s_{f_{1}(k+1)}\right)$ intersects $I\left(s_{f_{1}(k)}\right)$ or $I\left(s_{f_{1}(k+1)}\right)$ is to the right of $I\left(s_{f_{1}(k)}\right)$. If the former case happens, we do not move $s_{f_{1}(k+1)}$; otherwise, we move $s_{f_{1}(k+1)}$ leftwards until its left extension is at the right extension of $s_{f_{1}(k)}$. The above procedure stops once all sensors of $Q_{1}^{\prime}$ have been considered. We have the following observations. First, each sensor of $Q_{1}^{\prime}$ either does not change its position or has been moved 
leftwards. Second, sensors of $Q_{1}^{\prime}$ in $C^{\prime}$ together cover a continuous interval, denoted by $I\left(Q_{1}^{\prime}\right)$, whose left endpoint is $\alpha^{\prime}$. Further, due to the above first observation, it holds that $I\left(Q_{1}^{\prime}\right) \subseteq \mathcal{I}^{\prime}=\left[\alpha^{\prime}, \beta^{\prime}\right]$. Third, note that sensors of $Q_{1}^{\prime}$ may cover multiple disjoint maximal sub-intervals of $\mathcal{I}^{\prime}$ in $C$. Our above way of moving sensors of $Q_{1}^{\prime}$ leftwards guarantees that the length of $I\left(Q_{1}^{\prime}\right)$ is larger than or equal to the sum of the lengths of the above sub-intervals of $\mathcal{I}^{\prime}$ covered by sensors of $Q_{1}^{\prime}$ in $C$.

In the following, we show that each sensor of $Q_{1}^{\prime}$ is valid in $C^{\prime}$. Consider any sensor $s_{k} \in Q_{1}^{\prime}$. It is sufficient to show that $x_{k}\left(C^{\prime}\right) \in\left[x_{k}^{\prime}-2 \lambda, x_{k}^{\prime}\right]$.

Due to the above first observation, $x_{k}\left(C^{\prime}\right) \leq x_{k}(C)$. Since $s_{k}$ is valid in $C, x_{k}(C) \leq x_{k}^{\prime}$. Thus, we have $x_{k}\left(C^{\prime}\right) \leq x_{k}^{\prime}$. Next, we show that $x_{k}\left(C^{\prime}\right) \geq x_{k}^{\prime}-2 \lambda$.

On the one hand, since $s_{k} \in Q_{1}^{\prime}$, the left extension of $s_{k}$ is smaller than the left extension of $s_{f(j)}$ in $C_{0}$. On the other hand, the left extension of $s_{f(j)}$ is at $\alpha^{\prime}$ in $C$ while the left extension of $s_{k}$ is at least $\alpha^{\prime}$ in $C^{\prime}$. This implies the leftward moving distance of $s_{k}$ from $x_{k}^{\prime}$ in $C_{0}$ to $x_{k}\left(C^{\prime}\right)$ in $C^{\prime}$ is smaller than the leftward moving distance of $s_{f(j)}$ from $x_{f(j)}^{\prime}$ in $C_{0}$ to $x_{f(j)}(C)$ in $C$. Formally, $x_{k}^{\prime}-x_{k}\left(C^{\prime}\right) \leq x_{f(j)}^{\prime}-x_{f(j)}(C)$. Since $s_{f(j)}$ is valid in $C$, we have $x_{f(j)}^{\prime}-x_{f(j)}(C) \leq 2 \lambda$. Hence, we obtain $x_{k}^{\prime}(C) \geq x_{k}^{\prime}-2 \lambda$.

This proves that $s_{k}$ is valid in $C^{\prime}$.

Let $\beta_{1}^{\prime}$ denote the right endpoint of $I\left(Q_{1}^{\prime}\right)$. Thus, $I\left(Q_{1}^{\prime}\right)=\left[\alpha^{\prime}, \beta_{1}^{\prime}\right]$.

2. We consider the sensor $s_{t}$.

We move $s_{t}$ to a new location $x_{t}\left(C^{\prime}\right)$ as follows. Since the right extension of $s_{t}$ is at $\beta^{\prime}$ and $I\left(Q_{1}^{\prime}\right) \subseteq \mathcal{I}^{\prime}$, either $I\left(s_{t}\right)$ intersects $I\left(Q_{1}^{\prime}\right)$ or $I\left(s_{t}\right)$ is to the right of $I\left(Q_{1}^{\prime}\right)$.

If the former case happens, the interval $\mathcal{I}^{\prime}=\left[\alpha^{\prime}, \beta^{\prime}\right]$ is currently covered by sensors in $Q_{1}^{\prime}$ and $s_{t}$. Hence, we do not need to move the sensors of $Q_{2}^{\prime} \cup\left\{s_{f(j)}\right\}$ any more. We then proceed to apply the same argument on the sequence of sensors $s_{f(0)}, s_{f(1)}, \ldots, s_{f(j-1)}$ followed by sensors in $Q_{1}^{\prime}$ in the configuration $C^{\prime}$. Note that $s_{f(j)}$ is not in the above sequence of sensors.

Below, we assume $I\left(s_{t}\right)$ is to the right of $I\left(Q_{1}^{\prime}\right)$. We move $s_{t}$ leftwards until its left extension is at $\beta_{1}^{\prime}$. In this way, sensors in $Q_{1}^{\prime}$ and $s_{t}$ together cover the interval $\left[\alpha^{\prime}, \beta_{1}^{\prime}+2 r_{t}\right]$ in $C^{\prime}$. Note that $s_{t}$ is valid in $C^{\prime}$. The proof is similar to that in the normal sensor case and we omit it.

3. We consider the sensor $s_{f(j)}$.

By the definition of $\mathcal{I}^{\prime}=\left[\alpha^{\prime}, \beta^{\prime}\right]$, the right extension of $s_{f(j)}$ is to the left of $\beta^{\prime}$. We move $s_{f(j)}$ rightwards such that its right extension is at $\beta^{\prime}$. Below, we show that $s_{f(j)}$ is still valid in $C^{\prime}$. It is sufficient to show that $s_{f(j)}\left(C^{\prime}\right) \in\left[x_{f(j)}^{\prime}-2 \lambda, x_{f(j)}^{\prime}\right]$. First of all, since $s_{f(j)}$ is valid in $C$ and we have moved it rightwards to $x_{f(j)}\left(C^{\prime}\right)$, it holds that $x_{f(j)}\left(C^{\prime}\right) \geq x_{f(j)}(C) \geq x_{f(j)}^{\prime}-2 \lambda$. Next, we show that $x_{f(j)}\left(C^{\prime}\right) \leq x_{f(j)}^{\prime}$.

On the one hand, since $s_{f(j)}$ is a normal sensor, its right extension is larger than that of $s_{t}$ in $C_{0}$. On the other hand, the right extension of $s_{f(j)}$ in $C^{\prime}$ is at $\beta^{\prime}$, which is the same as the right extension of $s_{t}$ in $C$. This implies that comparing with its location $x_{f(j)}^{\prime}$ in $C_{0}$, $s_{f(j)}$ must have been moved leftwards to reach $x_{f(j)}\left(C^{\prime}\right)$ in $C^{\prime}$. In other words, it holds that $x_{f(j)}\left(C^{\prime}\right) \leq x_{f(j)}^{\prime}$.

This shows that $s_{f(j)}$ is still valid in $C^{\prime}$. 
4. Finally, we consider the sensors of $Q_{2}^{\prime}$.

Recall that currently in $C^{\prime}$, sensors of $Q_{1}^{\prime}$ together cover the interval $I\left(Q_{1}^{\prime}\right)=\left[\alpha^{\prime}, \beta_{1}^{\prime}\right], s_{t}$ covers $\left[\beta_{1}^{\prime}, \beta_{1}^{\prime}+2 r_{t}\right]$, and sensor $s_{f(j)}$ covers $\left[\beta^{\prime}-2 r_{f(j)}, \beta^{\prime}\right]$.

If $\beta_{1}^{\prime}+2 r_{t} \geq \beta^{\prime}-2 r_{f(j)}$, then $\mathcal{I}^{\prime}=\left[\alpha^{\prime}, \beta^{\prime}\right]$ is covered by sensors of $Q_{1}^{\prime} \cup\left\{s_{t}, s_{f(j)}\right\}$. In this cae, we do not need to move any sensor of $Q_{2}^{\prime}$.

In the following, we assume $\beta_{1}^{\prime}+2 r_{t}<\beta^{\prime}-2 r_{f(j)}$. We claim that the length of the interval $\left[\beta_{1}^{\prime}+2 r_{t}, \beta^{\prime}-2 r_{f(j)}\right]$ is no larger than the sum of the lengths of the covering intervals of all sensors in $Q_{2}^{\prime}$. Indeed, we have shown above the the length of $I\left(Q_{1}^{\prime}\right)$ is no smaller than the sum of the sub-invervals of $\mathcal{I}^{\prime}$ covered by the sensors of $Q_{1}^{\prime}$ in $C$. On the other hand, in $C^{\prime}$ the three intervals $I\left(Q_{1}^{\prime}\right), I\left(s_{t}\right)$, and $I\left(s_{f(j)}\right)$ are pairwise disjoint execept at their endpoints. Since $\mathcal{I}^{\prime}$ are covered by all sensors of $Q_{1}^{\prime} \cup Q_{2}^{\prime} \cup\left\{s_{t}, s_{f(j)}\right\}$ in $C$. The claim must hold.

In the sequel, we give a moving algorithm to move sensors of $Q_{2}^{\prime}$ to cover the interval $\left[\beta_{1}^{\prime}+\right.$ $\left.2 r_{t}, \beta^{\prime}-2 r_{f(j)}\right]$ such that every sensor of $Q_{2}^{\prime}$ is still valid.

Initially, let $I$ refer to the covering interval $I\left(s_{f(j)}\right)$, and we use $\alpha(I)$ to denote the left endpoint of $I$. Thus, $I=\left[\alpha(I), \beta^{\prime}\right]$. During the algorithm we will update $I$ and $\alpha(I)$. Let $I^{\prime}=\left[\alpha^{\prime}, \beta_{1}^{\prime}+2 r_{t}\right]$. Note that initially $\beta_{1}^{\prime}+2 r_{t}$ is less than $\alpha(I)$. We take an arbitrary sensor $s_{k}$ from $Q_{2}^{\prime}$ and remove it from $Q_{2}^{\prime}$. We do the following on $s_{k}$.

We move $s_{k}$ such that the right extension of $s_{k}$ is at $\alpha(I)$. In the following, we show that $s_{k}$ is valid in $C^{\prime}$ (i.e., after the above movement). Our goal is to show that $x_{k}\left(C^{\prime}\right) \in\left[x_{k}^{\prime}-2 \lambda, x_{k}^{\prime}\right]$.

On the one hand, since $s_{k}$ is a special sensor, the right extension of $s_{k}$ is smaller than the right extension of $s_{t}$ in $C_{0}$. In $C^{\prime}$, the right extension of $s_{k}$ (i.e., $\left.\alpha(I)\right)$ is larger than the right extension of $s_{t}$ (i.e., $\beta_{1}^{\prime}+2 r_{t}$ ). This means that the left moving distance of $s_{t}$ from $x_{t}^{\prime}$ in $C_{0}$ to $x_{t}\left(C^{\prime}\right)$ in $C^{\prime}$ is larger than the left moving distance of $s_{k}$ from $x_{k}^{\prime}$ in $C_{0}$ to $x_{k}\left(C^{\prime}\right)$ in $C^{\prime}$. Since we have proved above that $s_{t}$ is valid in $C^{\prime}$, we conclude that the left moving distance of $s_{k}$ from $x_{k}^{\prime}$ in $C_{0}$ to $x_{k}\left(C^{\prime}\right)$ in $C^{\prime}$ is no more than $2 \lambda$, i.e., $x_{k}\left(C^{\prime}\right) \geq x_{k}^{\prime}-2 \lambda$.

On the other hand, since $s_{k}$ is from $Q_{2}^{\prime}$, the right extension of $s_{k}$ is larger than or equal to the left extension of $s_{f(j)}$ in $C_{0}$. However, in $C^{\prime}$, the right extension of $s_{k}$ is smaller than or equal to the left extension of $s_{f(j)}$. This means that comparing with its location $x_{k}^{\prime}$ in $C_{0}, s_{k}$ must have been moved leftwards to reach $x_{k}\left(C^{\prime}\right)$ in $C^{\prime}$. In other words, it holds that $x_{k}\left(C^{\prime}\right) \leq x_{k}^{\prime}$.

This proves that $s_{k}$ is still valid in $C^{\prime}$.

Next we check whether $I\left(s_{k}\right)$ now intersects $I^{\prime}$. If yes, then $\mathcal{I}^{\prime} \subseteq I^{\prime} \cup I\left(s_{k}\right) \cup I$, which means that $\mathcal{I}^{\prime}$ is now covered in $C^{\prime}$. Hence, we stop the moving algorithm. Otherwise, we update $I=I\left(s_{k}\right) \cup I$ and $\alpha(I)=\alpha(I)-2 r_{k}$. We proceed to take another sensor of $Q_{2}^{\prime}$ and apply the same moving algorithm as above.

Due to our previous claim that the length of the interval $\left[\beta_{1}^{\prime}+2 r_{t}, \beta^{\prime}-2 r_{f(j)}\right]$ is no larger than the sum of the lengths of the covering intervals of all sensors in $Q_{2}^{\prime}$. The above moving algorithm will eventually stop and $\mathcal{I}^{\prime}$ will be covered in $C^{\prime}$. The above also shows that every sensor is valid in $C^{\prime}$.

Next, we continue to apply the similar argument on the new sequence of sensors $s_{f(1)}, s_{f(2)}, \ldots, s_{f(j-1)}$ followed by sensors in $Q_{1}^{\prime}$ and $s_{t}$ in the new configuration $C^{\prime}$. Note that $s_{f(j)}$ is not in the above sequence.

The above shows that in either case (i.e., $s_{f(m)}$ is a normal or special sensor) we can always move $s_{t}$ leftwards to "switch" with a normal sensor. We keep doing the same thing on $s_{t}$ and the 
remaining sequence of sensors until $s_{h}$ is considered, in which case $s_{t}$ will be moved to $y_{t}$ (note that $Q_{1}^{\prime}=\emptyset$ when $s_{h}$ is considered since $s_{h}$ is in $S_{12}$ ).

This completes the proof of the base case, i.e., there is always a configuration $C_{1}^{\prime}$ in which the interval $\left[0, x^{\prime}\right]$ is covered by the sensors of $S_{i}^{\prime}$ and the position of the sensor $s_{g(1)}$ in $C_{1}^{\prime}$ is $y_{g(1)}$.

We assume inductively that the claim holds for each $k-1$ with $2 \leq k \leq i$, i.e., there is a configuration $C_{k-1}^{\prime}$ in which the interval $\left[0, x^{\prime}\right]$ is covered by the sensors of $S_{i}^{\prime}$ and the position of the sensor $s_{g(j)}$ for each $1 \leq j \leq k-1$ in $C_{k-1}^{\prime}$ is $y_{g(j)}$. In the following, we show that the claim holds for $k$, i.e., there is a configuration $C_{k}^{\prime}$ in which the interval $\left[0, x^{\prime}\right]$ is still covered by the sensors of $S_{i}^{\prime}$ and the position of the sensor $s_{g(j)}$ for each $1 \leq j \leq k$ in $C_{k}^{\prime}$ is $y_{g(j)}$. The proof is quite similar to that for the base case and we only discuss it briefly below.

Let $t=g(k)$. If the position of $s_{t}$ in $C_{k-1}^{\prime}$ is $y_{t}$, then we are done (with $C_{k}^{\prime}=C_{k-1}^{\prime}$ ). Otherwise, let $y_{t}^{\prime}$ be the position of $s_{t}$ in $C_{k-1}^{\prime}$, with $y_{t}^{\prime} \neq y_{t}$. Depending on $s_{t} \in S_{k 1}$ or $s_{t} \in S_{k 2}$, there are two cases.

- If $s_{t} \in S_{k 1}$, then $y_{t}=x_{t}^{\prime}$. Since $y_{t}$ is the rightmost position to which $s_{t}$ is allowed to move and $y_{t}^{\prime} \neq y_{t}$, we have $y_{t}^{\prime}<y_{t}$. Depending on whether $s_{t}$ is in the critical set $S_{C}$, there further are two subcases.

If $s_{t} \notin S_{C}$, then the sensors in $S_{C}$ always form a coverage of $\left[0, x^{\prime}\right]$ regardless of where $s_{t}$ is. Thus, if we move $s_{t}$ to $y_{t}$, we obtain a new configuration $C_{k}^{\prime}$ from $C_{k-1}^{\prime}$ in which the sensors of $S_{i}^{\prime}$ still form a coverage of $\left[0, x^{\prime}\right]$ and the position of the sensor $s_{g(j)}$ for each $1 \leq j \leq k$ in $C_{k}^{\prime}$ is $y_{g(j)}$.

If $s_{t} \in S_{C}$, then since $y_{t}>y_{t}^{\prime}$, if we move $s_{t}$ from $y_{t}^{\prime}$ to $y_{t}, s_{t}$ is moved to the right. By Lemma 1(c), the interval $\left[0, R_{k-1}\right]$ is covered by the sensors of $S_{k-1}=\left\{s_{g(1)}, s_{g(2)}, \ldots, s_{g(k-1)}\right\}$ in $C_{k-1}^{\prime}$ (since they are in positions $y_{g(1)}, y_{g(2)}, \ldots, y_{g(k-1)}$, respectively). When $s_{t}$ is at $y_{t}, s_{t}$ still covers the point $p^{+}\left(R_{k-1}\right)$. Thus, after moving $s_{t}$ to $y_{t}$, we obtain a new configuration $C_{k}^{\prime}$ from $C_{k-1}^{\prime}$ in which the sensors of $S_{i}^{\prime}$ still form a coverage of $\left[0, x^{\prime}\right]$.

- If $s_{t} \in S_{k 2}$, then $S_{k 1}=\emptyset$ in this case, and $s_{t}$ is the sensor in $S_{k 2}$ with the smallest right extension. If $s_{t} \notin S_{C}$, then by the same argument as above, we can obtain a configuration $C_{k}^{\prime}$ from $C_{k-1}^{\prime}$ in which the interval $\left[0, x^{\prime}\right]$ is still covered by the sensors of $S_{i}^{\prime}$ and the position of the sensor $s_{g(j)}$ for each $1 \leq j \leq k$ in $C_{k}^{\prime}$ is $y_{g(j)}$. Below, we discuss the case when $s_{t} \in S_{C}$.

In $S_{C}$, some sensors must cover the point $p^{+}\left(R_{k-1}\right)$ in $C$. Let $S^{\prime}$ be the set of sensors in $S_{C}$ that cover $p^{+}\left(R_{k-1}\right)$ in $C$. If $s_{t} \in S^{\prime}$, then $y_{t}^{\prime}<y_{t}$ since $y_{t}$ is the rightmost position for $s_{t}$ to cover $p^{+}\left(R_{k-1}\right)$. In this case, again, by the same argument as above, we can move $s_{t}$ to the right from $y_{t}^{\prime}$ to $y_{t}$ to obtain a configuration $C_{k}^{\prime}$ from $C_{k-1}^{\prime}$ in which the interval $\left[0, x^{\prime}\right]$ is still covered by the sensors of $S_{i}^{\prime}$. Otherwise (i.e., $s_{t} \notin S^{\prime}$ ), consider a sensor $s_{h}$ in $S^{\prime}$. Let the sensors in $S_{C}$ between $s_{h}$ and $s_{t}$ in the cover order be $s_{h}, s_{f(1)}, s_{f(2)}, \ldots, s_{f(m)}, s_{t}$ (this sequence may contain only $s_{h}$ and $s_{t}$ ). Note that for each $1 \leq j \leq k-1$, the sensor $s_{g(j)}$ is not in this sequence. Then by using a similar sequence of switch operations as for the base case, we can obtain a new configuration $C_{k}^{\prime}$ from $C_{k-1}^{\prime}$ such that the sensors of $S_{i}^{\prime}$ still form a coverage of $\left[0, x^{\prime}\right]$. Again, the position of the sensor $s_{g(j)}$ for each $1 \leq j \leq k$ in $C_{k}^{\prime}$ is $y_{g(j)}$.

This proves that the claim holds for $k$. Therefore, the claim is true. The lemma can then be easily proved by using this claim, as follows.

Suppose the largest left-aligned interval that can be covered by the sensors of $S_{i}^{\prime}$ is $\left[0, x^{\prime}\right]$. Then by the above claim, there always exists a configuration $C^{*}$ for $S_{i}^{\prime}$ in which the interval $\left[0, x^{\prime}\right]$ is also 
covered by the sensors of $S_{i}^{\prime}$ and for each $1 \leq j \leq i$, the position of the sensor $s_{g(j)}$ in $C^{*}$ is $y_{g(j)}$. Recall that $S_{i}=\left\{s_{g(1)}, s_{g(2)}, \ldots, s_{g(i)}\right\}$. Then for each sensor $s_{t} \in S_{i}^{\prime} \backslash S_{i}$, the rightmost point that can be covered by $s_{t}$ is $x_{t}^{\prime}+r_{t}$. Recall that in the configuration $C_{i}$, for each $1 \leq j \leq i$, the position of the sensor $s_{g(j)}$ is $y_{g(j)}$, and for each sensor $s_{t} \in S_{i}^{\prime} \backslash S_{i}$, the position of $s_{t}$ is $x_{t}^{\prime}$. Further, by the definition of $S_{i}^{\prime}$, the right extensions of all sensors in $S_{i}^{\prime}$ are at most $R_{i}$ in $C_{i}$. Therefore, the right extensions of all sensors in $S_{i}^{\prime}$ are also at most $R_{i}$ in $C^{*}$, implying that $x^{\prime} \leq R_{i}$. On the other hand, by Lemma 1(c), the sensors of $S_{i}$ form a coverage of $\left[0, R_{i}\right]$ in $C^{*}$. Thus, $\left[0, x^{\prime}\right]=\left[0, R_{i}\right]$, and the lemma follows.

Finally, we prove the correctness of our algorithm based on Lemma 2. Suppose our algorithm reports $\lambda<\lambda^{*}$ in step $i$. Then according to the algorithm, $R_{i-1}<L$ and both $S_{i 1}$ and $S_{i 2}$ are $\emptyset$. Let $S_{i-1}^{\prime}$ be the set of sensors whose right extensions are at most $R_{i-1}$ in $C_{i-1}$. Since both $S_{i 1}$ and $S_{i 2}$ are $\emptyset$, no sensor in $S \backslash S_{i-1}^{\prime}$ can cover any point to the left of the point $p^{+}\left(R_{i-1}\right)$ (and including $\left.p^{+}\left(R_{i-1}\right)\right)$. By Lemma 2, [0, $\left.R_{i-1}\right]$ is the largest left-aligned interval that can be covered by the sensors of $S_{i-1}^{\prime}$. Hence, the sensors in $S$ cannot cover the interval $\left[0, p^{+}\left(R_{i-1}\right)\right]$. Due to $R_{i-1}<L$, we have $\left[0, p^{+}\left(R_{i-1}\right)\right] \subseteq[0, L]$; thus the sensors of $S$ cannot cover $B=[0, L]$. In other words, there is no feasible solution for the distance $\lambda$. This establishes the correctness of our algorithm.

\subsubsection{The Algorithm Implementation}

For the implementation of the algorithm, we first discuss a straightforward approach that runs in $O(n \log n)$ time. Later, we give another approach which, after $O(n \log n)$ time preprocessing, can determine whether $\lambda^{*} \leq \lambda$ in $O(n)$ time for any given $\lambda$. Although the second approach does not change the overall running time of our decision algorithm, it does help our optimization algorithm in Section 2.2 to run faster.

In the beginning, we sort the $2 n$ extensions of all sensors by the $x$-coordinate, and move each sensor $s_{i} \in S$ to $x_{i}^{\prime}$ to produce the initial configuration $C_{0}$. During the algorithm, for each step $i$, we maintain two sets of sensors, $S_{i 1}$ and $S_{i 2}$, as defined earlier. To this end, we sweep along the $x$-axis and maintain $S_{i 1}$ and $S_{i 2}$, using two sweeping points $p_{1}$ and $p_{2}$, respectively. Specifically, the point $p_{1}$ follows the positions $R_{0}(=0), R_{1}, R_{2}, \ldots$, and $p_{2}$ follows the positions $R_{0}+2 \lambda, R_{1}+2 \lambda, R_{2}+2 \lambda, \ldots$. Thus, $p_{2}$ is kept always by a distance of $2 \lambda$ to the right of $p_{1}$. To maintain the set $S_{i 1}$, when the sweeping point $p_{1}$ encounters the left extension of a sensor, we insert the sensor into $S_{i 1}$; when $p_{1}$ encounters the right extension of a sensor, we delete the sensor from $S_{i 1}$. In this way, when the sweeping point $p_{1}$ is at $R_{i-1}$, we have the set $S_{i 1}$ ready. To maintain $S_{i 2}$, the situation is slightly more subtle. First, whenever the sweeping point $p_{2}$ encounters the left extension of a sensor, we insert the sensor into $S_{i 2}$. The subtle part is at the deletion operation. By the definition of $S_{i 2}$, if the left extension of any sensor is less than or equal to $R_{i-1}$, then it should not be in $S_{i 2}$. Since eventually the first sweeping point $p_{1}$ is at $R_{i-1}$ in step $i$, whenever a sensor is inserted into the first set $S_{i 1}$, we need to delete that sensor from $S_{i 2}$. Thus, a deletion on $S_{i 2}$ happens only when the same sensor is inserted into $S_{i 1}$. In addition, we need a search operation on $S_{i 1}$ for finding the sensor in $S_{i 1}$ with the largest right extension, and a search operation on $S_{i 2}$ for finding the sensor in $S_{i 2}$ with the smallest right extension.

It is easy to see that there are $O(n)$ insertions and deletions in the entire algorithm. Further, the search operations on both $S_{i 1}$ and $S_{i 2}$ are dependent on the right extensions of the senors. By using a balanced binary search tree to represent each of these two sets in which the right extensions of the sensors are used as keys, the algorithm runs in $O(n \log n)$ time.

In the sequel, we give the second approach which, after $O(n \log n)$ time preprocessing, can determine whether $\lambda^{*} \leq \lambda$ in $O(n)$ time for any given $\lambda$. 


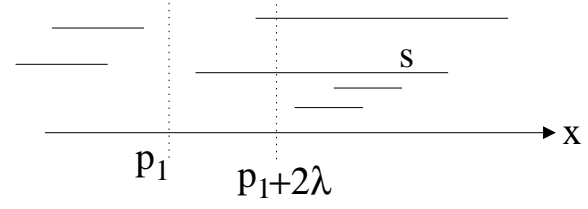

Figure 7: Illustrating the search for the sensor $s$ : the sensors before $p_{1}$ are removed from $S_{R}$; the two sensors below $s$ are redundant sensors for this step.

In the preprocessing, we compute two sorted lists $S_{L}$ and $S_{R}$, where $S_{L}$ contains all sensors sorted by the increasing values of their left extensions and $S_{R}$ contains all sensors sorted by the increasing values of their right extensions. Consider any value $\lambda$. Later in the algorithm, for each step $i$, our algorithm will determine the sensor $s_{g(i)}$ by scanning the two lists. We will show that when the algorithm finishes, each sensor in $S_{L}$ is scanned at most once and each sensor in $S_{R}$ is scanned at most three times, and therefore, the algorithm runs in $O(n)$ time.

Initially, we move each sensor $s_{i} \in S$ to $x_{i}^{\prime}$ to produce the initial configuration $C_{0}$. During the algorithm, we sweep along the $x$-axis, using a sweeping point $p_{1}$. Specifically, the point $p_{1}$ follows the positions $R_{0}(=0), R_{1}, R_{2}, \ldots$. With a little abuse of notation, we also let $p_{1}$ be the coordinate of the current position of $p_{1}$. Initially, $p_{1}=0$.

Consider a general step $i$ and we need to determine the sensor $s_{g(i)}$. In the beginning of this step, $p_{1}$ is at the position $R_{i-1}$. We scan the list $S_{L}$ from the beginning until the left extension of the next sensor is strictly to the right of $p_{1}$. For each scanned sensor $s_{j}$, if its right extension is strictly to the right of $p_{1}$, then it is in $S_{i 1}$ by the definition of $S_{i 1}$. Thus, the above scanning procedure can determine $S_{i 1}$, after which we can easily find the sensor $s_{g(i)}$ in $S_{i 1}$ if $S_{i 1} \neq \emptyset$. In fact, we can compute $s_{g(i)}$ directly in the above scanning procedure. In addition, for any sensor in $S_{L}$ that is scanned, we remove it from $S_{L}$ (and thus it will never be scanned later in the algorithm any more). If $S_{i 1} \neq \emptyset$, then $s_{g(i)}$ is determined and we move the sweeping point $p_{1}$ to the right extension of $s_{g(i)}$ (i.e., $p_{1}=R_{i}$ ). If $p_{1} \geq L$, we terminate the algorithm and report $\lambda^{*} \leq \lambda$; otherwise, we continue on to step $i+1$. Below, we discuss the case $S_{i 1}=\emptyset$.

If $S_{i 1}=\emptyset$, then $s_{g(i)}$ is the sensor in $S_{i 2}$ with the smallest right extension if $S_{i 2} \neq \emptyset$. Specifically, among the sensors (if any) whose left extensions are larger than $p_{1}\left(=R_{i-1}\right)$ and at most $p_{1}+2 \lambda$, $s_{g(i)}$ is the sensor with the smallest right extension. To find $s_{g(i)}$, we scan the list $S_{R}$ from the beginning until we find the first sensor $s$ whose left extension is larger than $p_{1}$ and at most $p_{1}+2 \lambda$ (see Fig. 7). If such a sensor $s$ does not exist, then $S_{i 2}=\emptyset$, and we terminate the algorithm and report $\lambda^{*}>\lambda$. Below, we assume we have found such a sensor $s$. Since the sensors in $S_{R}$ are sorted by their right extensions, $s_{g(i)}$ is exactly the sensor $s$. Further, unlike the scanning on $S_{L}$ where each scanned sensor is removed immediately, for each scanned sensor in $S_{R}$, we remove it only if its right extension is to the left of $p_{1}$ (see Fig. 7). Specifically, when we are searching the above sensor $s$ during scanning $S_{R}$, we remove from $S_{R}$ those sensors whose right extensions are to the left of $p_{1}$. It is easy to see that the removed sensors (if any) are consecutive from the beginning of $S_{R}$. Let $S_{R}$ be the list after all removals. If $s_{g(i)}(=s)$ is not the first sensor in $S_{R}$, then for any sensor $s_{j}$ in $S_{R}$ before $s_{g(i)}$, the left extension of $s_{j}$ must be larger than $p_{1}+2 \lambda$; we call the sensors in $S_{R}$ before $s_{g(i)}$ the redundant sensors for the step $i$ (see Fig. 7). Later we will show that these sensors will be not redundant any more in the later algorithm. In summary, for each sensor scanned in the original $S_{R}$ in this step, it is removed, or a redundant sensor, or $s_{g(i)}$. Finally, we move $s_{g(i)}$ to the left such that its left extension is at $p_{1}$, and then we move $p_{1}$ to the right extension of $s_{g(i)}$ (i.e., $p_{1}=R_{i}$ ). If $p_{1} \geq L$, we terminate the algorithm and report $\lambda^{*} \leq \lambda$; otherwise, we continue on the next step $i+1$. 
To analyze the algorithm, it is easy to see each sensor in this list $S_{L}$ is scanned at most once. For the list $S_{R}$, this may not be the case as the redundant sensors may be scanned again in the later algorithm. However, the following lemma shows that this would not be an issue.

Lemma 3 If a sensor $s_{j}$ is a redundant sensor for the step $i$, then it will be not a redundant sensor again in the later algorithm.

Proof: Consider the moment right after the step $i$. The sweeping point $p_{1}$ is at the right extension of $s_{g(i)}$. To prove the lemma, since $p_{1}$ always moves to the right, by the definition of the redundant sensors, it is sufficient to show that the left extension of $s_{j}$ is at most $p_{1}+2 \lambda$, as follows.

Consider the moment in the beginning of the step $i$ (the sensor $s_{g(i)}$ has not been moved to the left). Since $s_{j}$ is a redundant sensor for the step $i$, the sensor $s_{g(i)}$ is from $S_{i 2}$ and the left extension of $s_{g(i)}$ is at most $R_{i-1}+2 \lambda$. Thus, the right extension of $s_{g(i)}$ is at most $R_{i-1}+2 r_{g(i)}+2 \lambda$. Recall that the right extension of $s_{j}$ is less than that of $s_{g(i)}$ (since $s_{j}$ is before $s_{g(i)}$ in $S_{R}$ ). Therefore, the right extension of $s_{j}$ is at most $R_{i-1}+2 r_{g(i)}+2 \lambda$. Now consider the moment right after the step $i$. The sweeping point $p_{1}$ is at the position $R_{i-1}+2 r_{g(i)}$. Hence, the right extension of $s_{j}$ is at most $p_{1}+2 \lambda$, which implies that the left extension of $s_{j}$ is at most $p_{1}+2 \lambda$. The lemma thus follows.

The preceding lemma implies that any sensor can be a redundant sensor in at most one step. Therefore, for the list $S_{R}$, each sensor has been scanned at most twice when it is removed, once as a redundant sensor, and once when it is found as $s_{g(i)}$. Thus, each sensor in $S_{R}$ is scanned at most three times. Hence, after the two lists $S_{L}$ and $S_{R}$ are obtained, the running time of the algorithm is $O(n)$.

Theorem 1 After $O(n \log n)$ time preprocessing, for any $\lambda$, we can determine whether $\lambda^{*} \leq \lambda$ in $O(n)$ time; further, if $\lambda^{*} \leq \lambda$, we can compute a feasible solution in $O(n)$ time.

\subsubsection{Another Decision Version}

Our optimization algorithm in Section 2.2 also needs to determine whether $\lambda^{*}$ is strictly less than $\lambda$ (i.e., $\lambda^{*}<\lambda$ ) for any $\lambda$. By modifying our algorithm for Theorem 1, we have the following result.

Theorem 2 After $O(n \log n)$ time preprocessing, for any value $\lambda$, we can determine whether $\lambda^{*}<\lambda$ in $O(n)$ time.

Proof: We first apply the algorithm for Theorem 1 on the value $\lambda$. If the algorithm reports $\lambda^{*}>\lambda$, then we know $\lambda^{*}<\lambda$ is false. Otherwise, we have $\lambda^{*} \leq \lambda$. In the following, we modify the algorithm for Theorem 1 to determine whether $\lambda^{*}<\lambda$, i.e., $\lambda^{*}$ is strictly smaller than $\lambda$. Note that this is equivalent to deciding whether $\lambda^{*} \leq \lambda-\epsilon$ for any arbitrarily small constant $\epsilon>0$. Of course, we cannot enumerate all such small values $\epsilon$. Instead, we add a new mechanism to the algorithm for Theorem 1 such that the resulting displacement of each sensor is strictly smaller than $\lambda$.

At the start of the algorithm, we move all sensors to the right by a distance $\lambda$ to obtain the configuration $C_{0}$. But, the displacement of each sensor should be strictly less than $\lambda$. To ensure this, later in the algorithm, if the destination of a sensor $s_{i}$ is set as $y_{i}=x_{i}^{\prime}$, then we adjust this destination of $s_{i}$ by moving it to the left slightly such that $s_{i}$ 's displacement is strictly less than $\lambda$.

Consider a general step $i$ of the algorithm. We define the set $S_{i 1}$ in the same way as before, i.e., it consists of all sensors covering the point $p^{+}\left(R_{i-1}\right)$ in $C_{i-1}$. If $S_{i 1} \neq \emptyset$, then the algorithm is the same as before. In this case, the sensor $s_{g(i)}$ chosen in this step has a displacement of exactly $\lambda$, which is actually "illegal" since the displacement of each sensor should be strictly less than $\lambda$. We 
will address this issue later. However, if $S_{i 1}=\emptyset$, then the set $S_{i 2}$ is defined slightly different from before. Here, since $S_{i 1}=\emptyset$, we have to use a sensor to the right of $R_{i-1}$ in $C_{i-1}$ to cover $p^{+}\left(R_{i-1}\right)$. Since the displacement of each sensor should be strictly less than $\lambda$, we do not allow any sensor to move to the left by exactly the distance $2 \lambda$. To reflect this difference, we define $S_{i 2}$ as the set of sensors in $C_{i-1}$ each of which has its left extension larger than $R_{i-1}$ and strictly smaller than $R_{i-1}+2 \lambda$ (previously, it was "at most"). In this way, if we move a sensor in $S_{i 2}$ to the left to cover $p^{+}\left(R_{i-1}\right)$, then the displacement of that sensor is strictly less than $\lambda$. The rest of the algorithm is the same as before. We define the type I and type II sensors in the same way as before.

If the algorithm terminates without finding a feasible solution, then it must be $\lambda^{*} \geq \lambda$; otherwise, the algorithm finds a "feasible" solution $S O L$ with a critical set $S^{c}=\left\{s_{g(1)}, s_{g(2)}, \ldots, s_{g(m)}\right\}$. But, this does not necessarily mean $\lambda^{*}<\lambda$ since in $S O L$, the displacements of some sensors in $S^{c}$ may be exactly $\lambda$. Specifically, all type I sensors in $S^{c}$ are in the same positions as they are in $C_{0}$ and thus their displacements are exactly $\lambda$. In contrast, during the algorithm, the type II sensors in $S^{c}$ have been moved strictly to the left with respect to their positions in $C_{0}$; further, due to our new definition of the set $S_{i 2}$, the displacements of all type II sensors are strictly less than $\lambda$. Therefore, if there is no type I sensor in $S^{c}$, then the displacement of each sensor in $S^{c}$ is strictly less than $\lambda$ and thus we have $\lambda^{*}<\lambda$. Below we assume $S^{c}$ contains at least one type I sensor. To make sure that $\lambda^{*}<\lambda$ holds, we need to find a real feasible solution in which the displacement of each sensor in $S$ is strictly less than $\lambda$. On the other hand, to make sure that $\lambda^{*} \geq \lambda$ holds, we must show that there is no real feasible solution. For this, we apply the following algorithmic procedure.

We seek to adjust the solution $S O L$ to produce a real feasible solution. According to our algorithm, for each sensor $s_{i} \in S^{c}$, if it is a type I sensor, then $y_{i}=x_{i}^{\prime}$ and thus its displacement is exactly $\lambda$; otherwise, its displacement is less than $\lambda$. The purpose of our adjustment of $S O L$ is to move all type I sensors slightly to the left so that (1) their displacements are strictly less than $\lambda$, and (2) we can still form a coverage of $B$. In certain cases, we may need to use some sensors in $S \backslash S^{c}$ as well. Also, we may end up with the conclusion that no real feasible solution exists.

According to our algorithm, after finding the last sensor $s_{g(m)}$ in $S^{c}$, we have $R_{m} \geq L$. If $R_{m}>L$, then we can always adjust $S O L$ to obtain a real feasible solution by shifting each sensor in $S^{c}$ to the left by a very small value $\epsilon$ such that (1) the resulting displacement of each sensor in $S^{c}$ is less than $\lambda$, and (2) the sensors of $S^{c}$ still form a coverage of $B$. Note that there always exists such a small value $\epsilon$ such that the above adjustment is possible. Therefore, if $R_{m}>L$, then we have $\lambda^{*}<\lambda$.

If $R_{m}=L$, however, then the above strategy does not work. There are two cases. If there is a sensor $s_{t} \in S \backslash S^{c}$ such that $x_{t} \in\left(L-\lambda-r_{t}, L+\lambda+r_{t}\right)$, then we can also obtain a real feasible solution by shifting the sensors of $S^{c}$ slightly to the left as above and using the sensor $s_{t}$ to cover the remaining part of $B$ around $L$ that is no longer covered by the shifted sensors of $S^{c}$; thus we also have $\lambda^{*}<\lambda$. Otherwise, we claim that it must be $\lambda^{*} \geq \lambda$. Below we prove this claim.

Consider the rightmost Type I sensor $s_{i}$ in $S^{c}$. Suppose $s_{i}=s_{g(j)}$, i.e., $s_{i}$ is determined in step $j$. Thus, $s_{i}$ is at $x_{i}^{\prime}$ in $S O L$. Let $\epsilon>0$ be an arbitrarily small value (we will determine below how small it should be). Since we have assumed that the extensions of all sensors are different, the value $\epsilon$ can be made small enough such that by moving $s_{i}$ to $x_{i}^{\prime}-\epsilon$ in $C_{0}$, the relative order of the extensions of all sensors remains the same as before. Further, according to our algorithm above, the value $\epsilon$ can also be small enough such that the behavior of the algorithm is the same as before, i.e., the algorithm finds the same critical set $S^{c}$ with the same cover order as before. It is easy to see that such a small value $\epsilon$ always exists. Note that our task here is to prove our claim $\lambda^{*} \geq \lambda$ is true, and knowing that such a value $\epsilon$ exists is sufficient for our purpose and we need not actually 
find such a value $\epsilon$ in our algorithm.

Now, in step $j$, the new value $R_{j}$, which is the right extension of $s_{i}$, is $\epsilon$ smaller than its value before since $s_{i}$ was at $x_{i}^{\prime}$ in $C_{0}$. Because $s_{i}$ is the rightmost type I sensor in $S^{c}$, after step $j$, all sensors in $S^{c}$ determined after $s_{i}$ (if any) are of type II and thus are moved to the left such that they are all in attached positions along with $s_{i}$, which implies that the right extension of the last sensor $s_{g(m)}$ in $S^{c}$ is also $\epsilon$ smaller than its previous value (which was $L$ ). Hence, after step $m$, the sensors in $S_{m}$ covers $[0, L-\epsilon]$. As discussed above, if $\epsilon$ is made small enough, the behavior of the algorithm is the same as before. By a similar analysis, we can also establish a result similar to Lemma 2. Namely, $[0, L-\epsilon]$ is the largest left-aligned interval that can be covered by the sensors in $S_{m}^{\prime}$ in this setting (here, $S_{m}^{\prime}$ is the set of sensors whose right extensions are at most $L-\epsilon$ in the configuration after step $m$ ). We omit the detailed analysis for this, which is very similar to that for Lemma 2. Note that $S^{c}=S_{m}$. Since there is no sensor $s_{t} \in S \backslash S^{c}$ such that $x_{t} \in\left(L-\lambda-r_{t}, L+\lambda+r_{t}\right)$, the interval $(L-\epsilon, L]$ cannot be fully covered by the sensors in $S$. The above discussion implies that if we do not allow the displacement of $s_{i}$ to be larger than $\lambda-\epsilon$, then there would be no feasible solution even if we allow the displacements of some other sensors (i.e., those type I sensors in $S^{c}$ before $s_{i}$, if any) to be larger than $\lambda-\epsilon$ (but at most $\lambda$ ). Thus, $\lambda^{*} \leq \lambda-\epsilon$ cannot be true. That is, $\lambda^{*}>\lambda-\epsilon$ holds. Further, it is easy to see that, by a similar argument, for any fixed value $\epsilon^{\prime}>0$ with $\epsilon^{\prime}<\epsilon$, we also have $\lambda^{*}>\lambda-\epsilon^{\prime}$. Hence, we obtain $\lambda^{*} \geq \lambda$.

This finishes the discussion on how to determine whether $\lambda^{*}<\lambda$. It is easy to see that the above algorithm can also be implemented in $O(n)$ time for each value $\lambda$, after $O(n \log n)$ time preprocessing. The theorem thus follows.

Theorems 1 and 2 together lead to the following corollary.

Corollary 1 After $O(n \log n)$ time preprocessing, for any value $\lambda$, we can determine whether $\lambda^{*}=$ $\lambda$ in $O(n)$ time.

\subsection{The Optimization Version of the General BCLS}

In this section, we discuss the optimization version of the general BCLS problem. We show that it is solvable in $O\left(n^{2} \log n\right)$ time, thus settling the open problem in 9 .

It should be pointed out that if we can determine a set $\Lambda$ of candidate values such that $\lambda^{*} \in \Lambda$, then we would use our decision algorithms given in Section 2.1 to find $\lambda^{*}$ in $\Lambda$. We will use this approach in Section 3 for the uniform case. However, so far it is not clear to us how to determine such a set $\Lambda$. Below, we use a different approach.

One main difficulty for solving the problem is that we do not know the order of the sensors in the optimal solution. Our strategy is to determine a critical set of sensors and their cover order in a feasible solution for the (unknown) optimal value $\lambda^{*}$. The idea is somewhat similar to parametric search [7, 15] and here we "parameterize" our algorithm for Theorem 1. But, unlike the typical parametric search [7, 15], our approach does not involve any parallel scheme and is practical. We first give an overview of this algorithm. In the following discussion, the "decision algorithm" refers to our algorithm for Theorem 1 unless otherwise stated.

Recall that given any value $\lambda$, step $i$ of our decision algorithm determines the sensor $s_{g(i)}$ and obtains the set $S_{i}=\left\{s_{g(1)}, s_{g(2)}, \ldots, s_{g(i)}\right\}$, in this order, which we also call the cover order of the sensors in $S_{i}$. In our optimization algorithm, we often use $\lambda$ as a variable. Thus, $S_{i}(\lambda)$ (resp., $R_{i}(\lambda)$, $s_{g(i)}(\lambda)$, and $\left.C_{i}(\lambda)\right)$ refers to the corresponding $S_{i}$ (resp., $R_{i}, s_{g(i)}$, and $C_{i}$ ) obtained by running our decision algorithm on the specific value $\lambda$. Denote by $C_{I}$ the configuration of the input. 


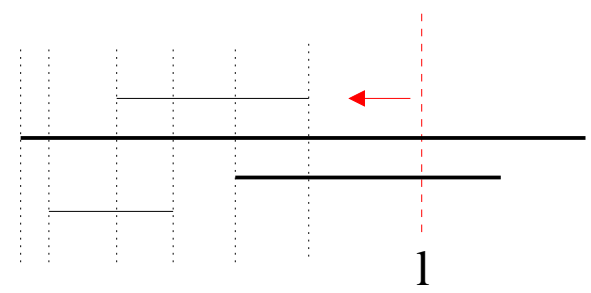

Figure 8: The vertical dashed line $l$ is $x=R_{i-1}(\lambda)$. The set $S_{i 1}(\lambda)$ consists of the sensors whose covering intervals intersect $l$, shown in bold. As $\lambda$ increases, the relative position of $l$ in $C_{i-1}(\lambda)$ moves to the left, and $S_{i 1}(\lambda)$ changes whenever $l$ hits a sensor extension, shown with the dotted vertical lines.

Our optimization algorithm takes at most $n$ steps. Initially, let $S_{0}\left(\lambda^{*}\right)=\emptyset, R_{0}\left(\lambda^{*}\right)=0, \lambda_{0}^{1}=0$, and $\lambda_{0}^{2}=+\infty$. For each $i \geq 1$, step $i$ receives an interval $\left(\lambda_{i-1}^{1}, \lambda_{i-1}^{2}\right)$ and a sensor set $S_{i-1}\left(\lambda^{*}\right)$, with the following algorithm invariants:

- $\lambda^{*} \in\left(\lambda_{i-1}^{1}, \lambda_{i-1}^{2}\right)$.

- For any value $\lambda \in\left(\lambda_{i-1}^{1}, \lambda_{i-1}^{2}\right), S_{i-1}(\lambda)=S_{i-1}\left(\lambda^{*}\right)$ and their cover orders are the same.

Step $i$ either finds the value $\lambda^{*}$ or determines a sensor $s_{g(i)}\left(\lambda^{*}\right)$. The interval $\left(\lambda_{i-1}^{1}, \lambda_{i-1}^{2}\right)$ will shrink to a new interval $\left(\lambda_{i}^{1}, \lambda_{i}^{2}\right) \subseteq\left(\lambda_{i-1}^{1}, \lambda_{i-1}^{2}\right)$ and we also obtain the set $S_{i}\left(\lambda^{*}\right)=S_{i-1}\left(\lambda^{*}\right) \cup$ $\left\{s_{g(i)}\left(\lambda^{*}\right)\right\}$. All these can be done in $O(n \log n)$ time. The details of the algorithm are given below.

Consider a general step $i$ for $i \geq 1$ and we have the interval $\left(\lambda_{i-1}^{1}, \lambda_{i-1}^{2}\right)$ and the set $S_{i-1}\left(\lambda^{*}\right)$. While discussing the algorithm, we will also prove inductively the following lemma about the function $R_{i}(\lambda)$ with variable $\lambda \in\left(\lambda_{i}^{1}, \lambda_{i}^{2}\right)$.

Lemma 4 For any step $i$ with $i \geq 0$, if the algorithm does not stop after the step, then the following hold:

(a) The function $R_{i}(\lambda)$ for $\lambda \in\left(\lambda_{i}^{1}, \lambda_{i}^{2}\right)$ is a line segment of slope 1 or 0 .

(b) We can compute the function $R_{i}(\lambda)$ for $\lambda \in\left(\lambda_{i}^{1}, \lambda_{i}^{2}\right)$ explicitly in $O(n)$ time.

(c) $R_{i}(\lambda)<L$ for any $\lambda \in\left(\lambda_{i}^{1}, \lambda_{i}^{2}\right)$.

In the base case for $i=0$, the statement of Lemma 4 obviously holds. We assume the lemma statement holds for $i-1$, in particular, the function $R_{i-1}(\lambda)$ for $\lambda \in\left(\lambda_{i-1}^{1}, \lambda_{i-1}^{2}\right)$ is already known. We will show that after step $i$ the lemma statement holds for $i$, and thus the lemma will be proved.

Again, in step $i$, we need to determine the sensor $s_{g(i)}\left(\lambda^{*}\right)$ and let $S_{i}\left(\lambda^{*}\right)=S_{i-1}\left(\lambda^{*}\right) \cup\left\{s_{g(i)}\left(\lambda^{*}\right)\right\}$. We will also obtain an interval $\left(\lambda_{i}^{1}, \lambda_{i}^{2}\right)$ such that $\lambda^{*} \in\left(\lambda_{i}^{1}, \lambda_{i}^{2}\right) \subseteq\left(\lambda_{i-1}^{1}, \lambda_{i-1}^{2}\right)$ and for any $\lambda \in$ $\left(\lambda_{i}^{1}, \lambda_{i}^{2}\right), S_{i}(\lambda)=S_{i}\left(\lambda^{*}\right)$ holds (with the same cover order).

To find the sensor $s_{g(i)}\left(\lambda^{*}\right)$, we first determine the set $S_{i 1}\left(\lambda^{*}\right)$. Recall that $S_{i 1}\left(\lambda^{*}\right)$ consists of all sensors covering the point $p^{+}\left(R_{i-1}\left(\lambda^{*}\right)\right)$ in the configuration $C_{i-1}\left(\lambda^{*}\right)$. For each sensor in $S \backslash S_{i-1}\left(\lambda^{*}\right)$, its position in the configuration $C_{i-1}(\lambda)$ with respect to $\lambda \in\left(\lambda_{i-1}^{1}, \lambda_{i-1}^{2}\right)$ is a function of slope 1. As $\lambda$ increases in $\left(\lambda_{i-1}^{1}, \lambda_{i-1}^{2}\right)$, by our assumption that Lemma 4 (a) holds for $i-1$, the function $R_{i-1}(\lambda)$ is a line segment of slope 1 or 0 . If $R_{i-1}(\lambda)$ is of slope 1 , then the relative position of $R_{i-1}(\lambda)$ in $C_{i-1}(\lambda)$ does not change and thus the set $S_{i 1}(\lambda)$ does not change; if the function $R_{i-1}(\lambda)$ is of slope 0 , then the relative position of $R_{i-1}(\lambda)$ in $C_{i-1}(\lambda)$ is monotonically moving to the left. Hence, there are $O(n)$ values for $\lambda$ in $\left(\lambda_{i-1}^{1}, \lambda_{i-1}^{2}\right)$ that can incur some changes to the set $S_{i 1}(\lambda)$ and each such value corresponds to a sensor extension (e.g., see Fig. 8); further, 
these values can be easily determined in $O(n \log n)$ time by a simple sweeping process (we omit the discussion of it). Let $\Lambda_{i 1}$ be the set of all these $\lambda$ values. Let $\Lambda_{i 1}$ also contain both $\lambda_{i-1}^{1}$ and $\lambda_{i-1}^{2}$, and thus, $\lambda_{i-1}^{1}$ and $\lambda_{i-1}^{2}$ are the smallest and largest values in $\Lambda_{i 1}$, respectively. We sort the values in $\Lambda_{i 1}$. For any two consecutive values $\lambda_{1}<\lambda_{2}$ in the sorted $\Lambda_{i 1}$, the set $S_{i 1}(\lambda)$ for any $\lambda \in\left(\lambda_{1}, \lambda_{2}\right)$ is the same. By using binary search on the sorted $\Lambda_{i 1}$ and our decision algorithm in Theorem 1, we determine (in $O(n \log n)$ time) the two consecutive values $\lambda_{1}$ and $\lambda_{2}$ in $\Lambda_{i 1}$ such that $\lambda_{1}<\lambda^{*} \leq \lambda_{2}$. Further, by Corollary 1, we determine whether $\lambda^{*}=\lambda_{2}$. If $\lambda^{*}=\lambda_{2}$, then we terminate the algorithm. Otherwise, based on our discussion above, $S_{i 1}\left(\lambda^{*}\right)=S_{i 1}(\lambda)$ for any $\lambda \in\left(\lambda_{1}, \lambda_{2}\right)$. Thus, to compute $S_{i 1}\left(\lambda^{*}\right)$, we can pick an arbitrary $\lambda$ in $\left(\lambda_{1}, \lambda_{2}\right)$ and find $S_{i 1}(\lambda)$ in the same way as in our decision algorithm. Hence, $S_{i 1}\left(\lambda^{*}\right)$ can be easily found in $O(n \log n)$ time. Note that $\lambda^{*} \in\left(\lambda_{1}, \lambda_{2}\right) \subseteq\left(\lambda_{i-1}^{1}, \lambda_{i-1}^{2}\right)$. Depending on whether $S_{i 1}\left(\lambda^{*}\right) \neq \emptyset$, there are two cases.

- If $S_{i 1}\left(\lambda^{*}\right) \neq \emptyset$, then $s_{g(i)}\left(\lambda^{*}\right)$ is the sensor in $S_{i 1}\left(\lambda^{*}\right)$ with the largest right extension. An obvious observation is that for any $\lambda \in\left(\lambda_{1}, \lambda_{2}\right)$, the sensor in $S_{i 1}(\lambda)$ with the largest right extension is the same, which can be easily found. We let $\lambda_{i}^{1}=\lambda_{1}$ and $\lambda_{i}^{2}=\lambda_{2}$. Let $S_{i}\left(\lambda^{*}\right)=S_{i-1}\left(\lambda^{*}\right) \cup\left\{s_{g(i)}\left(\lambda^{*}\right)\right\}$. The algorithm invariants hold. Further, as $\lambda$ increases in $\left(\lambda_{i}^{1}, \lambda_{i}^{2}\right)$, the right extension of $s_{g(i)}(\lambda)$, which is $R_{i}(\lambda)$, increases by the same amount. That is, the function $R_{i}(\lambda)$ on $\left(\lambda_{i}^{1}, \lambda_{i}^{2}\right)$ is a line segment of slope 1 . Therefore, we can compute $R_{i}(\lambda)$ on $\left(\lambda_{i}^{1}, \lambda_{i}^{2}\right)$ explicitly in constant time. This also shows Lemma 4 (a) and (b) hold for $i$.

- If $S_{i 1}\left(\lambda^{*}\right)=\emptyset$, then we need to compute $S_{i 2}\left(\lambda^{*}\right)$. For any $\lambda \in\left(\lambda_{1}, \lambda_{2}\right)$, the set $S_{i 2}(\lambda)$ consists of all sensors whose left extensions are larger than $R_{i-1}(\lambda)$ and at most $R_{i-1}(\lambda)+2 \lambda$ in the configuration $C_{i-1}(\lambda)$. Recall that the function $R_{i-1}(\lambda)$ on $\left(\lambda_{i-1}^{1}, \lambda_{i-1}^{2}\right)$ is linear with slope 1 or 0 . Due to $\left(\lambda_{1}, \lambda_{2}\right) \subseteq\left(\lambda_{i-1}^{1}, \lambda_{i-1}^{2}\right)$, the linear function $R_{i-1}(\lambda)+2 \lambda$ on $\left(\lambda_{1}, \lambda_{2}\right)$ is of slope 3 or 2. Again, as $\lambda$ increases, the position of each sensor in $S \backslash S_{i-1}\left(\lambda^{*}\right)$ in $C_{i-1}(\lambda)$ is a linear function of slope 1 . Hence, as $\lambda$ increases, the relative position of $R_{i-1}(\lambda)+2 \lambda$ in $C_{i-1}(\lambda)$ moves to the right, and the relative position of $R_{i-1}(\lambda)$ in $C_{i-1}(\lambda)$ either does not change or moves to the left. Therefore, there are $O(n) \lambda$ values in $\left(\lambda_{1}, \lambda_{2}\right)$ each of which incurs some change to the set $S_{i 2}(\lambda)$ and each such $\lambda$ value corresponds to the left extension of a sensor (e.g., see Fig. 9). Further, these values can be easily determined in $O(n \log n)$ time by a sweeping process (we omit the discussion for this). (Actually, as $\lambda$ increases, the size of the set $S_{i 2}(\lambda)$ is monotonically increasing.) Let $\Lambda_{i 2}$ denote the set of these $\lambda$ values, and let $\Lambda_{i 2}$ contain both $\lambda_{1}$ and $\lambda_{2}$. Again, $\left|\Lambda_{i 2}\right|=O(n)$. We sort the values in $\Lambda_{i 2}$. Using binary search on the sorted $\Lambda_{i 2}$ and our decision algorithm in Theorem 1, we determine (in $O(n \log n)$ time) the two consecutive values $\lambda_{1}^{\prime}$ and $\lambda_{2}^{\prime}$ in $\Lambda_{i 2}$ such that $\lambda_{1}^{\prime}<\lambda^{*} \leq \lambda_{2}^{\prime}$. Further, by Corollary 1. we determine whether $\lambda^{*}=\lambda_{2}^{\prime}$. If $\lambda^{*}=\lambda_{2}^{\prime}$, then we are done. Otherwise, $S_{i 2}\left(\lambda^{*}\right)=S_{i 2}(\lambda)$ for any $\lambda \in\left(\lambda_{1}^{\prime}, \lambda_{2}^{\prime}\right)$, which can be easily found. Note that $\lambda^{*} \in\left(\lambda_{1}^{\prime}, \lambda_{2}^{\prime}\right) \subseteq\left(\lambda_{1}, \lambda_{2}\right)$.

The above obtains the set $S_{i 2}\left(\lambda^{*}\right)$. We claim that $S_{i 2}\left(\lambda^{*}\right) \neq \emptyset$. Indeed, due to our assumption that Lemma 4 holds for $i-1$, we have $R_{i-1}(\lambda)<L$ for $\lambda \in\left(\lambda_{i-1}^{1}, \lambda_{i-1}^{2}\right)$. Suppose to the contrary that $S_{i 2}\left(\lambda^{*}\right)=\emptyset$. Then, the sensor $s_{g(i)}\left(\lambda^{*}\right)$ does not exist, which implies that $S_{i-1}\left(\lambda^{*}\right)$ is the critical set for covering the barrier $B$ in an optimal solution. By our algorithm invariants, $\lambda^{*} \in\left(\lambda_{i-1}^{1}, \lambda_{i-1}^{2}\right)$ and $S_{i-1}\left(\lambda^{*}\right)$ is the same as $S_{i-1}(\lambda)$ for any $\lambda \in\left(\lambda_{i-1}^{1}, \lambda_{i-1}^{2}\right)$. Due to $R_{i-1}(\lambda)<L$ for $\lambda \in\left(\lambda_{i-1}^{1}, \lambda_{i-1}^{2}\right)$, the sensors in $S_{i-1}\left(\lambda^{*}\right)$ cannot cover the entire barrier $B$, which contradicts with that $S_{i-1}\left(\lambda^{*}\right)$ is the critical set in the optimal solution. Hence, $S_{i 2}\left(\lambda^{*}\right) \neq \emptyset$.

Since $S_{i 2}\left(\lambda^{*}\right) \neq \emptyset, s_{g(i)}\left(\lambda^{*}\right)$ is the sensor in $S_{i 2}\left(\lambda^{*}\right)$ with the smallest right extension. As before, the sensor in $S_{i 2}(\lambda)$ with the smallest right extension is the same for any $\lambda \in\left(\lambda_{1}^{\prime}, \lambda_{2}^{\prime}\right)$. 


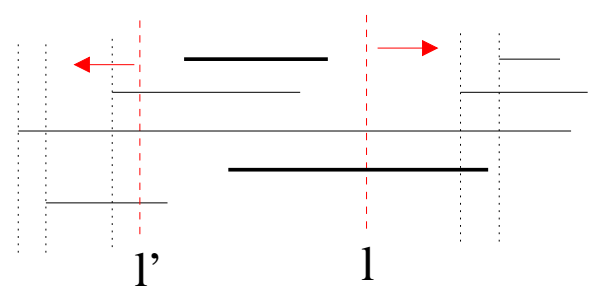

Figure 9: The two vertical dashed lines $l$ is $x=R_{i-1}(\lambda)+2 \lambda$ and $l^{\prime}$ is $x=R_{i-1}(\lambda)$. The set $S_{i 2}(\lambda)$ consists of sensors whose left extensions are between $l$ and $l^{\prime}$, shown in bold. As $\lambda$ increases, the relative position of $l$ in $C_{i-1}(\lambda)$ moves to the right, and the relative position of $l^{\prime}$ in $C_{i-1}(\lambda)$ either does not change or moves to the left. The set $S_{i 2}(\lambda)$ changes whenever $l$ or $l^{\prime}$ hits the left extension of a sensor, shown with the dotted vertical lines.

Thus, $s_{g(i)}\left(\lambda^{*}\right)$ can be easily determined. We let $\lambda_{i}^{1}=\lambda_{1}^{\prime}$ and $\lambda_{i}^{2}=\lambda_{2}^{\prime}$. Let $S_{i}\left(\lambda^{*}\right)=$ $S_{i-1}\left(\lambda^{*}\right) \cup\left\{s_{g(i)}\left(\lambda^{*}\right)\right\}$. The algorithm invariants hold. Further, we examine the function $R_{i}(\lambda)$, i.e., the right extension of $s_{g(i)}(\lambda)$ in the configuration $C_{i}(\lambda)$, as $\lambda$ increases in $\left(\lambda_{i}^{1}, \lambda_{i}^{2}\right)$. Since $s_{g(i-1)}\left(\lambda^{*}\right)$ and $s_{g(i)}\left(\lambda^{*}\right)$ are always in attached positions in this case, for any $\lambda \in\left(\lambda_{i}^{1}, \lambda_{i}^{2}\right)$, we have $R_{i}(\lambda)=R_{i-1}(\lambda)+2 r_{g(i)}$. Thus, the function $R_{i}(\lambda)$ is a vertical shift of $R_{i-1}(\lambda)$ by the distance $2 r_{g(i)}$. Because we already know explicitly the function $R_{i-1}(\lambda)$ for $\lambda \in\left(\lambda_{i}^{1}, \lambda_{i}^{2}\right)$, which is a line segment of slope 1 or 0 , the function $R_{i}(\lambda)$ can be computed in constant time, which is also a line segment of slope 1 or 0 . Note that this shows that Lemma 4 (a) and (b) hold for $i$.

If the algorithm does not stop, the above determines an interval $\left(\lambda_{i}^{1}, \lambda_{i}^{2}\right)$ such that the algorithm invariants and Lemma 4(a) and (b) hold on the interval. Below, we do further processing such that Lemma 4(c) also holds.

Because the function $R_{i}(\lambda)$ on $\left(\lambda_{i}^{1}, \lambda_{i}^{2}\right)$ is a line segment of slope 1 or 0 , there are three cases depending on the values $R_{i}(\lambda)$ and $L$ : (1) $R_{i}(\lambda)<L$ for any $\lambda \in\left(\lambda_{i}^{1}, \lambda_{i}^{2}\right),(2) R_{i}(\lambda)>L$ for any $\lambda \in\left(\lambda_{i}^{1}, \lambda_{i}^{2}\right)$, and (3) there exists $\lambda^{\prime} \in\left(\lambda_{i}^{1}, \lambda_{i}^{2}\right)$ such that $R_{i}\left(\lambda^{\prime}\right)=L$.

1. For Case (1), we proceed to the next step, along with the interval $\left(\lambda_{i}^{1}, \lambda_{i}^{2}\right)$. Clearly, the algorithm invariants hold and Lemma 4(c) holds for $i$.

2. For Case (2), the next lemma shows that it actually cannot happen due to $\lambda^{*} \in\left(\lambda_{i}^{1}, \lambda_{i}^{2}\right)$.

Lemma 5 It is not possible that $R_{i}(\lambda)>L$ for any $\lambda \in\left(\lambda_{i}^{1}, \lambda_{i}^{2}\right)$.

Proof: Assume to the contrary that $R_{i}(\lambda)>L$ for any $\lambda \in\left(\lambda_{i}^{1}, \lambda_{i}^{2}\right)$. Since $\lambda^{*} \in\left(\lambda_{i}^{1}, \lambda_{i}^{2}\right)$, let $\lambda^{\prime \prime}$ be any value in $\left(\lambda_{i}^{1}, \lambda^{*}\right)$. Due to $\lambda^{\prime \prime} \in\left(\lambda_{i}^{1}, \lambda_{i}^{2}\right)$, we have $R_{i}\left(\lambda^{\prime \prime}\right)>L$. But this would implies that we have found a feasible solution where the displacement of each sensor is at most $\lambda^{\prime \prime}$, which is smaller than $\lambda^{*}$, incurring contradiction.

3. For the Case (3), note that the slope of $R_{i}(\lambda)$ on $\left(\lambda_{i}^{1}, \lambda_{i}^{2}\right)$ can not be 0 . To see this, suppose to the contrary the slope of $R_{i}(\lambda)$ on $\left(\lambda_{i}^{1}, \lambda_{i}^{2}\right)$ is 0 . Then, $R_{i}(\lambda)=L$ for any $\lambda \in\left(\lambda_{i}^{1}, \lambda_{i}^{2}\right)$. Since $\lambda^{*} \in\left(\lambda_{i}^{1}, \lambda_{i}^{2}\right)$, for any $\lambda^{\prime} \in\left(\lambda_{i}^{1}, \lambda^{*}\right), R_{i}\left(\lambda^{\prime}\right)=L$, which means that there is a feasible solution where the displacement of each sensor is at most $\lambda^{\prime}<\lambda^{*}$, incurring contradiction.

Hence, $R_{i}(\lambda)$ on $\left(\lambda_{i}^{1}, \lambda_{i}^{2}\right)$ is a line segment of slope 1 , and thus we can determine in constant time the unique value $\lambda^{\prime} \in\left(\lambda_{i}^{1}, \lambda_{i}^{2}\right)$ such that $R_{i}\left(\lambda^{\prime}\right)=L$. Clearly, $\lambda^{*} \leq \lambda^{\prime}$. By Corollary 1, we determine whether $\lambda^{*}=\lambda^{\prime}$. If $\lambda^{*}=\lambda^{\prime}$, then we terminate the algorithm; otherwise, we have $\lambda^{*} \in\left(\lambda_{i}^{1}, \lambda^{\prime}\right)$ and update $\lambda_{i}^{2}$ to $\lambda^{\prime}$. We proceed to the next step, along with the interval $\left(\lambda_{i}^{1}, \lambda_{i}^{2}\right)$. Again, the algorithm invariants hold and Lemma 4(c) holds for $i$. 
This finishes the discussion of step $i$ of our algorithm. The running time of step $i$ is $O(n \log n)$. Note that in each case where we proceed to the next step, the statement of Lemma 4 holds for $i$, and thus Lemma 4 has been proved.

In the following lemma, we show that the algorithm must stop within at most $n$ steps.

Lemma 6 The algorithm finds $\lambda^{*}$ in at most $n$ steps.

Proof: Assume the critical set is $S_{k}\left(\lambda^{*}\right)$ for some $k$ if we run our decision algorithm with $\lambda=\lambda^{*}$. Since there are $n$ sensors from the input, we have $1 \leq k \leq n$.

We claim that our algorithm finds $\lambda^{*}$ in at most $k$ steps. Suppose to the contrary that the algorithm does not find $\lambda^{*}$ in the first $k$ steps. In other words, the algorithm does not stop after step $k$. By the algorithm invariants, after step $k$, we have an interval $\left(\lambda_{k}^{1}, \lambda_{k}^{2}\right)$ such that $\lambda^{*} \in\left(\lambda_{k}^{1}, \lambda_{k}^{2}\right)$ and $S_{k}(\lambda)=S_{k}\left(\lambda^{*}\right)$ for any $\lambda \in\left(\lambda_{k}^{1}, \lambda_{k}^{2}\right)$. Further, by Lemma $4, R_{k}(\lambda)<L$ for any $\lambda \in\left(\lambda_{k}^{1}, \lambda_{k}^{2}\right)$, which means that the sensors in $S_{k}\left(\lambda^{*}\right)$ cannot cover the entire barrier $B$ for any $\lambda \in\left(\lambda_{k}^{1}, \lambda_{k}^{2}\right)$, contradicting with that $S_{k}\left(\lambda^{*}\right)$ is the critical set for the decision algorithm when $\lambda=\lambda^{*}$.

Therefore, our algorithm finds $\lambda^{*}$ in at most $k$ steps. The lemma thus follows.

After $\lambda^{*}$ is found, by applying our decision algorithm on $\lambda=\lambda^{*}$, we finally produce an optimal solution in which the displacement of every sensor is at most $\lambda^{*}$. Since each step takes $O(n \log n)$ time, the total time of the algorithm is $O\left(n^{2} \log n\right)$.

Theorem 3 The general BCLS problem is solvable in $O\left(n^{2} \log n\right)$ time.

We shall make a technical remark. The typical parametric search [7, 15] usually returns with an interval containing the optimal value and then uses an additional step to find the optimal value. In contrast, our algorithm is guaranteed to find the optimal value $\lambda^{*}$ directly. This is due to the mechanism in our algorithm that requires $R_{i}(\lambda)<L$ for any $\lambda \in\left(\lambda_{i}^{1}, \lambda_{i}^{2}\right)$ after each step $i$ if the algorithm is not terminated. This mechanism actually plays the role of the additional step used in the typical parametric search.

\section{The Uniform Case of BCLS}

In this section, we present an $O(n \log n)$ time algorithm for the uniform case of BCLS. Previously, the best known algorithm for it takes $O\left(n^{2}\right)$ time [9]. Further, for the special uniform case when all sensors are initially located on the barrier $B$, we solve it in $O(n)$ time.

\subsection{Preliminaries}

Recall that in the input, all sensors are ordered from left to right by their initial positions, i.e., $x_{1} \leq x_{2} \leq \cdots \leq x_{n}$. Suppose in a solution, the destination of each sensor $s_{i}$ is $y_{i}(1 \leq i \leq n)$; then we say that the solution is order preserving if $y_{1} \leq y_{2} \leq \cdots \leq y_{n}$. In the uniform case, since all sensors have the same range, we let $r$ denote the sensor range. The next lemma was known 9 .

Lemma 7 (Czyzowicz et al. 9]) For the uniform case, there is always an optimal solution that is order preserving.

As discussed in [9], Lemma 7 is not applicable to the general BCLS. Consequently, the approach in this section does not work for the general BCLS. 
Based on the order preserving property in Lemma 7, the previous $O\left(n^{2}\right)$ time algorithm [9] tries to cover $B$ from left to right; each step picks the next sensor and re-balances the current maximum sensor movement. Here, we take a completely different approach.

Denote by $\lambda^{*}$ the maximum sensor movement in an optimal solution. We use OPT to denote an optimal order preserving solution in which the destination for each sensor $s_{i}$ is $y_{i}(1 \leq i \leq n)$. For each sensor $s_{i}$, if $x_{i}>y_{i}$ (resp., $x_{i}<y_{i}$ ), then we say $s_{i}$ is moved to the left (resp., right) by a distance $\left|x_{i}-y_{i}\right|$. A set of sensors is said to be in attached positions if the union of their covering intervals is a continuous interval on the $x$-axis whose length is equal to the sum of the lengths of the covering intervals of these sensors. A single sensor is always in attached position. The following lemma was proved in [9].

Lemma 8 (Czyzowicz et al. 9]) If $\lambda^{*}>0$, then in OPT, there exist a sequence of consecutive sensors $s_{i}, s_{i+1}, \ldots, s_{j}$ with $i \leq j$ such that they are in attached positions and one of the following three cases is true. (a) The sensor $s_{j}$ is moved to the left by the distance $\lambda^{*}$ and $y_{i}=r$ (i.e., the sensors $s_{i}, s_{i+1}, \ldots, s_{j}$ together cover exactly the interval $\left.[0,2 r(j-i+1)]\right)$. (b) The sensor $s_{i}$ is moved to the right by the distance $\lambda^{*}$ and $y_{j}=L-r$. (c) For $i \neq j$ (i.e., $i<j$ ), the sensor $s_{i}$ is moved to the right by the distance $\lambda^{*}$ and the sensor $s_{j}$ is moved to the left by the distance $\lambda^{*}$.

Cases (a) and (b) in Lemma 8 are symmetric. By Lemma 8, for each pair of sensors $s_{i}$ and $s_{j}$ with $i \leq j$, we can compute three distances $\lambda_{1}(i, j), \lambda_{2}(i, j)$, and $\lambda_{3}(i, j)$ corresponding to the three cases in Lemma 8 as candidates for the optimal distance $\lambda^{*}$. Specifically, $\lambda_{1}(i, j)=x_{j}-[2 r(j-i)+r]$, where the value $2 r(j-i)+r$ is supposed to be the destination of the sensor $s_{j}$ in OPT if case (a) holds. Symmetrically, $\lambda_{2}(i, j)=[L-2 r(j-i)-r]-x_{i}$. Let $\lambda_{3}(i, j)=\left[x_{j}-x_{i}-2 r(j-i)\right] / 2$ for $i<j$. Let $\Lambda$ be the set of all $\lambda_{1}(i, j), \lambda_{2}(i, j)$, and $\lambda_{3}(i, j)$ values. Clearly, $\lambda^{*} \in \Lambda$ and $|\Lambda|=\Theta\left(n^{2}\right)$. By using an algorithm for the decision version of the uniform case to search in $\Lambda$, one can find the value $\lambda^{*}$. Recall that the decision problem is that given any value $\lambda$, determine whether there exists a feasible solution for covering $B$ such that the moving distances of all sensors are at most $\lambda$. Thus, $\lambda^{*}$ is the smallest value in $\Lambda$ such that the answer to the decision problem on that value is "yes". A simple greedy $O(n)$ time algorithm was given in [9] for the decision problem.

Lemma 9 (Czyzowicz et al. [9]) The decision version of the uniform case is solvable in $O(n)$ time.

But, the above approach would take $\Omega\left(n^{2}\right)$ time due to $|\Lambda|=\Theta\left(n^{2}\right)$. To reduce the running time, we cannot compute the set $\Lambda$ explicitly. In general, our $O(n \log n)$ time algorithm uses the following idea. First, instead of computing all elements of $\Lambda$ explicitly, we compute one element of $\Lambda$ whenever we need it (we may do some preprocessing for this). Second, suppose we already know (implicitly) a sorted order of all values in $\Lambda$; then we can use binary search and the decision algorithm for Lemma 9 to find $\lambda^{*}$. However, we are not able to order the values of $\Lambda$ into a single sorted list; instead, we order them (implicitly) in $O(n)$ sorted lists and each list has $O(n)$ values. Consequently, by a technique called binary search in sorted arrays [6], we compute $\lambda^{*}$ in $O(n \log n)$ time. The details of our algorithm are given in the next subsection.

\subsection{Our Algorithm for the Uniform Case}

Due to the order preserving property, it is easy to check whether $\lambda^{*}=0$ in $O(n)$ time. In the following, we assume $\lambda^{*}>0$.

We focus on how to order (implicitly) the elements of $\Lambda$ into $O(n)$ sorted lists and each list contains $O(n)$ elements. We also show that after preprocessing, each element in any sorted list can be computed in $O(1)$ time using the index of the element. We aim to prove the next lemma. 
Lemma 10 In $O(n \log n)$ time, the elements of $\Lambda$ can be ordered (implicitly) into $O(n)$ sorted lists such that each list contains $O(n)$ elements and each element in any list can be computed in constant time by giving the index of the list and the index of the element in the list.

The following technique, called binary search on sorted arrays [6], will be applied. Suppose there is a "black-box" decision procedure $\Pi$ available such that given any value $a, \Pi$ can report whether $a$ is a feasible value to a certain problem in $O(T)$ time, and further, if $a$ is a feasible value, then any value larger than $a$ is also feasible. Given a set of $m$ arrays $A_{i}, 1 \leq i \leq m$, each containing $O(n)$ elements in sorted order, the goal is to find the smallest feasible value $\delta$ in $A=\cup_{i=1}^{m} A_{i}$. Suppose each element of any array can be obtained in constant time by giving its indices. An algorithm for the following result was presented by Chen et al. in [6].

Lemma 11 (Chen et al. [6]) The value $\delta$ in $A$ can be computed in $O((m+T) \log (n m))$ time.

If we use the algorithm for Lemma 9 as the decision procedure $\Pi$, then by Lemmas 10 and 11, we can find $\lambda^{*}$ in $\Lambda$ in $O(n \log n)$ time. After $\lambda^{*}$ is found, we can apply the algorithm for Lemma 9 to compute the destinations of all sensors, in $O(n)$ time. Hence, we have the following result.

Theorem 4 The uniform case of the BCLS problem is solvable in $O(n \log n)$ time.

In the rest of this subsection, we focus on proving Lemma 10 ,

For each $1 \leq t \leq 3$, let $\Lambda_{t}$ denote the set of all $\lambda_{t}(i, j)$ values. Clearly, $\Lambda=\Lambda_{1} \cup \Lambda_{2} \cup \Lambda_{3}$. We seek to order each of the three sets $\Lambda_{1}, \Lambda_{2}$, and $\Lambda_{3}$ into sorted lists.

We discuss $\Lambda_{1}$ first. This case is trivial. It is easy to see that for each given value $j$, we have $\lambda_{1}\left(i_{1}, j\right) \leq \lambda_{1}\left(i_{2}, j\right)$ for any $i_{1} \leq i_{2} \leq j$. Thus, for every value $j$, we have a sorted list $\lambda_{1}(1, j), \lambda_{1}(2, j), \ldots, \lambda_{1}(j, j)$ of $j$ elements, and each element can be computed in constant time by using the index of the element in the list. Therefore, we have $n$ sorted lists, and clearly, the set of elements in all these lists is exactly $\Lambda_{1}$. Hence we have the following lemma.

Lemma 12 In $O(n \log n)$ time, the elements of $\Lambda_{1}$ can be ordered (implicitly) into $O(n)$ sorted lists such that each list contains $O(n)$ elements and each element in any list can be computed in constant time by giving the index of the list and the index of the element in the list.

The set $\Lambda_{2}$ can be processed in a symmetric manner as $\Lambda_{1}$, and we omit the details.

Lemma 13 In $O(n \log n)$ time, the elements of $\Lambda_{2}$ can be ordered (implicitly) into $O(n)$ sorted lists such that each list contains $O(n)$ elements and each element in any list can be computed in constant time by giving the index of the list and the index of the element in the list.

In the following, we focus on ordering (implicitly) the set $\Lambda_{3}$ and showing the following lemma, which, together with Lemmas 12 and 13, proves Lemma 10.

Lemma 14 In $O(n \log n)$ time, the elements of $\Lambda_{3}$ can be ordered (implicitly) into $O(n)$ sorted lists such that each list contains $O(n)$ elements and each element in any list can be computed in constant time by giving the index of the list and the index of the element in the list. 


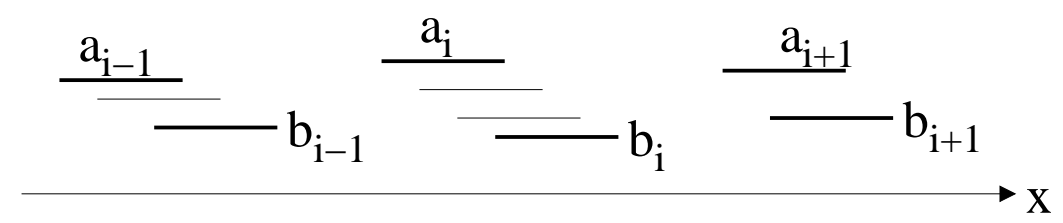

Figure 10: Illustrating three groups of sensors: $G_{i-1}, G_{i}$, and $G_{i+1}$. The sensors with indices $a_{i-1}, a_{i}$, and $a_{i+1}$ (resp., $b_{i-1}, b_{i}$, and $b_{i+1}$ ) are in $S_{a}$ (resp., $S_{b}$ ), which are indicated by thick segments.

Proving Lemma 14 is a main challenge to our uniform case algorithm. The reason is that, unlike $\Lambda_{1}$ and $\Lambda_{2}$, for a given $j$, for any $1 \leq i_{1} \leq i_{2} \leq j$, either $\lambda_{3}\left(i_{1}, j\right) \leq \lambda_{3}\left(i_{2}, j\right)$ or $\lambda_{3}\left(i_{1}, j\right) \geq \lambda_{3}\left(i_{2}, j\right)$ is possible. Hence, to prove Lemma 14, we have to find another way to order the elements of $\Lambda_{3}$.

Our approach is to first remove some elements from $\Lambda_{3}$ that are surely not $\lambda^{*}$ (for example, negative values cannot be $\lambda^{*}$ ). We begin with some intuitions. We say two intervals on the $x$-axis are strictly overlapped if they contain more than one common point. In the following discussion, the sensors are always at their input positions unless otherwise stated. We define two subsets of sensors, $S_{a}$ and $S_{b}$, as follows. A sensor $s_{j}$ is in $S_{a}$ if and only if there is no sensor $s_{i}$ with $i<j$ such that their covering intervals are strictly overlapped (e.g., see Fig. 10). A sensor $s_{i}$ is in $S_{b}$ if and only if there is no sensor $s_{j}$ with $i<j$ such that their covering intervals are strictly overlapped. Let the indices of sensors in $S_{a}$ be $a_{1}, a_{2}, \ldots, a_{n_{1}}$ and the indices of sensors in $S_{b}$ be $b_{1}, b_{2}, \ldots, b_{n_{2}}$, from left to right. We claim $n_{1}=n_{2}$. To see this, consider the interval graph $G$ in which the covering interval of each sensor is a vertex and two vertices are connected by an edge if their corresponding intervals are strictly overlapped. Observe that in each connected component of $G$, there is exactly one interval whose corresponding sensor is in $S_{a}$ and there is exactly one interval whose corresponding sensor is in $S_{b}$, and vice versa. Thus, $n_{1}=n_{2}$, which is the number of connected components of $G$. Let $m=n_{1}=n_{2} \leq n$. Further, it is easy to see that the covering intervals of both $a_{i}$ and $b_{i}$ must be in the same connected component of $G$ and $a_{i} \leq b_{i}$. Indeed, $a_{i}$ (resp., $b_{i}$ ) is the leftmost (resp., rightmost) sensor in the subset of sensors whose covering intervals are in the same connected component of $G$ (see Fig. 10). Note that $a_{i}=b_{i}$ is possible. Hence, $G$ has $m$ connected components.

For each $1 \leq i \leq m$, let $G_{i}$ denote the connected component containing the covering intervals of $a_{i}$ and $b_{i}$; with a little abuse of notation, we also use $G_{i}$ to denote the subset of sensors whose covering intervals are in the connected component $G_{i}$. Clearly, $G_{i}=\left\{s_{j} \mid a_{i} \leq j \leq b_{i}\right\}$ (e.g., see Fig. 10). We also call $G_{i}$ a group of sensors. The groups $G_{1}, G_{2}, \ldots, G_{m}$ form a partition of $S$. The sensor $s_{a_{i}}$ (resp., $s_{b_{i}}$ ) is the leftmost (resp., rightmost) sensor in $G_{i}$.

Lemma 15 For any two sensors $s_{i}$ and $s_{j}$ with $i<j$, if $s_{i} \notin S_{b}$ or $s_{j} \notin S_{a}$, then $\lambda_{3}(i, j) \neq \lambda^{*}$.

Proof: Assume $s_{i} \notin S_{b}$. In the following, we prove that $\lambda_{3}(i, j)$ cannot be $\lambda^{*}$ for any $i<j$.

Suppose $s_{i}$ is in the group $G_{k}$. Then $i<b_{k}$ due to $s_{i} \notin S_{b}$. Further, the covering intervals of $s_{i}$ and $s_{i+1}$ must be strictly overlapped (otherwise, $s_{i}$ would be in $S_{b}$ ). Assume to the contrary $\lambda_{3}(i, j)=\lambda^{*}$, which implies that case (c) in Lemma 8 holds. Thus, in the corresponding $O P T, s_{i}$ is moved to the right by the distance $\lambda_{3}(i, j)$ and all sensors $s_{i}, s_{i+1}, \ldots, s_{j}$ must be in attached positions. It is easy to see that the sensor $s_{i+1}$ must move to the right by the distance $\lambda_{3}(i, j)+2 r-$ $\left(x_{i+1}-x_{i}\right)$. Since the covering intervals of $s_{i}$ and $s_{i+1}$ are strictly overlapped, $2 r-\left(x_{i+1}-x_{i}\right)>0$. Therefore, the moving distance of $s_{i+1}$ must be larger than that of $s_{i}$. Since the moving distance of $s_{i}$ is $\lambda_{3}(i, j)=\lambda^{*}$, we have contradiction. Hence, $\lambda_{3}(i, j)$ cannot be $\lambda^{*}$.

Assume $s_{j} \notin S_{a}$. Then by a symmetric argument, we can prove $\lambda_{3}(i, j) \neq \lambda^{*}$ for any $i<j$. 
By Lemma 15, if $\lambda^{*} \in \Lambda_{3}$, then it can only be in the set $\Lambda_{3}^{\prime}=\left\{\lambda_{3}(i, j) \mid i<j, i \in S_{b}, j \in S_{a}\right\}$, and $\left|\Lambda_{3}^{\prime}\right|=O\left(m^{2}\right)$. Thus, we seek to order the elements of $\Lambda_{3}^{\prime}$ into $O(m)$ sorted lists and each list contains $O(m)$ elements. One might be tempting to use the following way. Clearly, for each $1 \leq k \leq m-1, \Lambda_{3}^{\prime}$ contains $\lambda_{3}\left(s_{b_{k}}, s_{a_{h}}\right)$ for all $h=k+1, k+2, \ldots, m$, and hence one may simply put them into a list. However, such a list is not necessarily sorted. Specifically, for any two indices $h_{1}$ and $h_{2}$ with $k+1 \leq h_{1}<h_{2} \leq m$, either $\lambda_{3}\left(s_{b_{k}}, s_{a_{h_{1}}}\right) \leq \lambda_{3}\left(s_{b_{k}}, s_{a_{h_{2}}}\right)$ or $\lambda_{3}\left(s_{b_{k}}, s_{a_{h_{1}}}\right)>\lambda_{3}\left(s_{b_{k}}, s_{a_{h_{2}}}\right)$ is possible. Our approach relies on additional observations. Below, for simplicity of notation, we use $\lambda_{3}\left(b_{k}, a_{h}\right)$ to refer to $\lambda_{3}\left(s_{b_{k}}, s_{a_{h}}\right)$. We first examine the value of each $\lambda_{3}\left(b_{k}, a_{h}\right)$ in $\Lambda_{3}^{\prime}$.

By definition, we have $\lambda_{3}\left(b_{k}, a_{k+1}\right)=\left(x_{a_{k+1}}-x_{b_{k}}-2 r\right) / 2$, and this is equal to half the length of the interval between the right extension of $s_{b_{k}}$ and the left extension of $s_{a_{k+1}}$, which we call a gap. Note that this gap is $\emptyset$ when the two sensors $s_{b_{k}}$ and $s_{a_{k+1}}$ are in attached positions. For each $1 \leq k \leq m-1$, define $g_{k}=x_{a_{k+1}}-x_{b_{k}}-2 r$, which is the length of the corresponding gap. Hence, $\lambda_{3}\left(b_{k}, a_{k+1}\right)=g_{k} / 2$. Further, for each $1 \leq k \leq m$, we define the width of the group $G_{k}$ as the length of the union interval of the covering intervals of the sensors in $G_{k}$, and define $l_{k}$ as the sum of the lengths of the covering intervals of the sensors in $G_{k}$ minus the width of $G_{k}$, i.e., $l_{k}$ is equal to $2 r\left(b_{k}-a_{k}+1\right)$ minus the width of $G_{k}$. We then have the following observation.

Observation 3 For every $k$ with $1 \leq k \leq m-2$, we have $\lambda_{3}\left(b_{k}, a_{h}\right)=\left(\sum_{t=k}^{h-1} g_{t}-\sum_{t=k+1}^{h-1} l_{t}\right) / 2$ for each $h$ with $k+2 \leq h \leq m$.

Proof: By definition, $\lambda_{3}\left(b_{k}, a_{h}\right)=\left[\left(x_{a_{h}}-x_{b_{k}}-2 r\right)-2 r\left(a_{h}-b_{k}-1\right)\right] / 2$. It is easy to see that the value $x_{a_{h}}-x_{b_{k}}-2 r$ is equal to $\sum_{t=k}^{h-1} g_{t}$ plus the sum of the widths of all groups $G_{k+1}, G_{k+2}, \ldots, G_{h-1}$, and the value $2 r\left(a_{h}-b_{k}-1\right)$ is equal to the sum of the lengths of the covering intervals of the sensors in the union of the groups $G_{k+1}, G_{k+2}, \ldots, G_{h-1}$. According to definitions of $l_{t}$ for $k+1 \leq t \leq h-1$, the observation follows.

The following lemma will be useful later.

Lemma 16 For four indices $k_{1}, k_{2}, h_{1}$, and $h_{2}$, suppose $\max \left\{k_{1}, k_{2}\right\}<\min \left\{h_{1}, h_{2}\right\}$; then $\lambda_{3}\left(b_{k_{1}}, a_{h_{1}}\right)$ $-\lambda_{3}\left(b_{k_{1}}, a_{h_{2}}\right)=\lambda_{3}\left(b_{k_{2}}, a_{h_{1}}\right)-\lambda_{3}\left(b_{k_{2}}, a_{h_{2}}\right)$, and consequently, $\lambda_{3}\left(b_{k_{1}}, a_{h_{1}}\right) \leq \lambda_{3}\left(b_{k_{1}}, a_{h_{2}}\right)$ if and only if $\lambda_{3}\left(b_{k_{2}}, a_{h_{1}}\right) \leq \lambda_{3}\left(b_{k_{2}}, a_{h_{2}}\right)$.

Proof: Note that for every $1 \leq k \leq m-2$, we have $\lambda_{3}\left(b_{k}, a_{h}\right)=\left(\sum_{t=k}^{h-1} g_{t}-\sum_{t=k+1}^{h-1} l_{t}\right) / 2$ for $k+2 \leq h \leq m$, and $\lambda_{3}\left(b_{k}, a_{h}\right)=g_{k} / 2$ for $h=k+1$.

If $h_{1}=h_{2}$, then the lemma trivially follows since $\lambda_{3}\left(b_{k_{1}}, a_{h_{1}}\right)=\lambda_{3}\left(b_{k_{1}}, a_{h_{2}}\right)$ and $\lambda_{3}\left(b_{k_{2}}, a_{h_{1}}\right)=$ $\lambda_{3}\left(b_{k_{2}}, a_{h_{2}}\right)$. Thus we consider $h_{1} \neq h_{2}$, and only show the case with $h_{1}<h_{2}$ (the case with $h_{1}>h_{2}$ is similar). By their definitions, we have $\lambda_{3}\left(b_{k_{1}}, a_{h_{1}}\right)-\lambda_{3}\left(b_{k_{1}}, a_{h_{2}}\right)=\left(-\sum_{t=h_{1}}^{h_{2}-1} g_{t}+\sum_{t=h_{1}}^{h_{2}-1} l_{t}\right) / 2$. Similarly, $\lambda_{3}\left(b_{k_{2}}, a_{h_{1}}\right)-\lambda_{3}\left(b_{k_{2}}, a_{h_{2}}\right)=\left(-\sum_{t=h_{1}}^{h_{2}-1} g_{t}+\sum_{t=h_{1}}^{h_{2}-1} l_{t}\right) / 2$. Hence, the lemma follows.

Lemma[16]implies that for any $k_{1}$ and $k_{2}$ with $1 \leq k_{1}<k_{2} \leq m-1$, the sorted order of $\lambda_{3}\left(b_{k_{1}}, a_{t}\right)$ for all $t=k_{2}+1, k_{2}+2, \ldots, m$ is the same as that of the list $\lambda_{3}\left(b_{k_{2}}, a_{t}\right)$ for $t=k_{2}+1, k_{2}+2, \ldots, m$ in terms of the indices of $a_{t}$. This means that if we sort the values in the list $\lambda_{3}\left(b_{1}, a_{t}\right)$ for all $t=2,3, \ldots, m$, then for any $1<k \leq m-1$, the sorted order of the list $\lambda_{3}\left(b_{k}, a_{t}\right)$ with all $t=k+1, k+2, \ldots, m$ is also obtained implicitly. Our "ordering" algorithm works as follows.

We first explicitly compute the values $\lambda_{3}\left(b_{1}, a_{t}\right)$ for all $t=2,3, \ldots, m$, which takes $O(m)$ time, and then sort them in $O(m \log m)$ time. Let $p$ be the permutation of $2,3, \ldots, m$ such that the increasing sorted list of these $\lambda_{3}\left(b_{1}, a_{t}\right)$ values is $\lambda_{3}\left(b_{1}, a_{p(1)}\right), \lambda_{3}\left(b_{1}, a_{p(2)}\right), \ldots, \lambda_{3}\left(b_{1}, a_{p(m-1)}\right)$. Note that the permutation $p$ is immediately available once we obtain the above sorted list. For any $1<k<m$, we say the element $\lambda_{3}\left(b_{k}, a_{h}\right)$ is valid if $k+1 \leq h \leq m$ and is undefined otherwise. 
By Lemma 16, the valid elements in each list $\lambda_{3}\left(b_{k}, a_{p(1)}\right), \lambda_{3}\left(b_{k}, a_{p(2)}\right), \ldots, \lambda_{3}\left(b_{k}, a_{p(m-1)}\right)$ are also sorted increasingly. Further, if we compute $g_{1}, g_{2}, \ldots, g_{m-1}$ and $l_{1}, l_{2}, \ldots, l_{m}$ as well as their prefix sums in the preprocessing, then given the index of any valid element in the list, we can obtain its actual value in $O(1)$ time. Clearly, the preprocessing takes $O(n \log n)$ time. Thus, we have ordered (implicitly) the elements of $\Lambda_{3}^{\prime}$ into $O(m)$ sorted lists and each list has $O(m)$ elements.

However, we are not done yet. Since eventually we will apply the binary search technique of Lemma 11 to these sorted lists and the lists contain undefined elements, the algorithm may take an undefined element in such a list and use it in the decision procedure which is the algorithm for Lemma 9. But, the undefined elements do not have meaningful values. To resolve this, we assign (implicitly) to each undefined element an "appropriate" value, as follows. For each $1 \leq k<m$, let $\mathcal{L}(k)$ denote the list $\lambda_{3}\left(b_{k}, a_{p(1)}\right), \lambda_{3}\left(b_{k}, a_{p(2)}\right), \ldots, \lambda_{3}\left(b_{k}, a_{p(m-1)}\right)$. If $1<k<m$, then the list $\mathcal{L}(k)$ has some undefined elements. For each undefined element, we (implicitly) assign an actual value to it such that the resulting new list is still sorted. The idea is inspired by Lemma 16. We use the list $\mathcal{L}(1)$ as the reference list since all its elements are valid. Every other list $\mathcal{L}(k)$ has at least one valid element, for example, the element $\lambda_{3}\left(b_{k}, a_{k+1}\right)$. We compute explicitly the value $\lambda_{3}\left(b_{k}, a_{k+1}\right)$ for each $1<k<m$, in $O(m)$ time. For a list $\mathcal{L}(k)$ with $1<k<m$ and any undefined element $\lambda_{3}\left(b_{k}, a_{p(i)}\right)$ in $\mathcal{L}(k)$, we assign to it (implicitly) the value $\lambda_{3}\left(b_{k}, a_{k+1}\right)+\lambda_{3}\left(b_{1}, a_{p(i)}\right)-\lambda_{3}\left(b_{1}, a_{k+1}\right)$ (note that all these three values have already been computed explicitly). The lemma below shows that the resulting new list $\mathcal{L}(k)$ is still sorted increasingly with this value assignment scheme.

Lemma 17 For any $1<k<m$, the list $\mathcal{L}(k)$ is still sorted increasingly after all its undefined elements are assigned values implicitly.

Proof: Consider any $k$ with $1<k<m$, and any two indices $i$ and $j$ with $1 \leq i<j \leq m-1$. It is sufficient to prove $\lambda_{3}\left(b_{k}, a_{p(i)}\right) \leq \lambda_{3}\left(b_{k}, a_{p(j)}\right)$.

If both values are valid, then by Lemma 16, the inequality holds. Otherwise, we assume $\lambda_{3}\left(b_{k}, a_{p(i)}\right)$ is undefined. After our value assignment, $\lambda_{3}\left(b_{k}, a_{p(i)}\right)=\lambda_{3}\left(b_{k}, a_{k+1}\right)+\lambda_{3}\left(b_{1}, a_{p(i)}\right)-$ $\lambda_{3}\left(b_{1}, a_{k+1}\right)$. Depending on whether $\lambda_{3}\left(b_{k}, a_{p(j)}\right)$ is undefined, there are two cases.

If $\lambda_{3}\left(b_{k}, a_{p(j)}\right)$ is undefined, then we have $\lambda_{3}\left(b_{k}, a_{p(j)}\right)=\lambda_{3}\left(b_{k}, a_{k+1}\right)+\lambda_{3}\left(b_{1}, a_{p(j)}\right)-\lambda_{3}\left(b_{1}, a_{k+1}\right)$. Hence, $\lambda_{3}\left(b_{k}, a_{p(j)}\right)-\lambda_{3}\left(b_{k}, a_{p(i)}\right)=\lambda_{3}\left(b_{1}, a_{p(j)}\right)-\lambda_{3}\left(b_{1}, a_{p(i)}\right) \geq 0$ due to $j>i$. If $\lambda_{3}\left(b_{k}, a_{p(j)}\right)$ is valid, then by Lemma 16, we have $\lambda_{3}\left(b_{k}, a_{p(j)}\right)=\lambda_{3}\left(b_{k}, a_{k+1}\right)+\lambda_{3}\left(b_{1}, a_{p(j)}\right)-\lambda_{3}\left(b_{1}, a_{k+1}\right)$. Thus, $\lambda_{3}\left(b_{k}, a_{p(j)}\right)-\lambda_{3}\left(b_{k}, a_{p(i)}\right)=\lambda_{3}\left(b_{1}, a_{p(j)}\right)-\lambda_{3}\left(b_{1}, a_{p(i)}\right) \geq 0$.

Therefore, in both cases, we have $\lambda_{3}\left(b_{k}, a_{p(i)}\right) \leq \lambda_{3}\left(b_{k}, a_{p(j)}\right)$, which proves the lemma.

In summary, in $O(n \log n)$ time, we have (implicitly) ordered the elements of $\Lambda_{3}^{\prime}$ into $O(m)$ sorted lists and each list has $O(m)$ elements such that every element in any list can be obtained in $O(1)$ time. Hence, Lemma 14 is proved. We remark that assigning values to the undefined elements in $\Lambda_{3}^{\prime}$ as above does not affect the correctness of our algorithm. Assigning values to undefined elements only makes our candidate set $\Lambda$ for $\lambda^{*}$ a little larger (by a constant factor), which obviously does not affect the algorithm correctness because the larger candidate set still contains $\lambda^{*}$. One might also see that the statement of Lemma 14 (and thus Lemma 10) is a little imprecise since we actually ordered only the elements in a subset $\Lambda_{3}^{\prime}$ of $\Lambda_{3}$ (not the entire set $\Lambda_{3}$ ).

\subsection{The Special Uniform Case}

In this subsection, we consider the special uniform case in which all sensors are initially located on the barrier $B=[0, L]$, i.e., $0 \leq x_{i} \leq L$ for each $1 \leq i \leq n$. We give an $O(n)$ time algorithm for it. Again, we assume $\lambda^{*}>0$. 
Clearly, Lemmas 7 and 8 still hold. Further, since all sensors are initially on $B$, in case (a) of Lemma 8, $s_{i}$ must be $s_{1}$. To see this, since $s_{1}$ is initially located on $B=[0, L]$, it is always the best to use $s_{1}$ to cover the beginning portion of $B$ due to the order preserving property. We omit the formal proof of this. Similarly, in case (b) of Lemma 8, $s_{j}$ must be $s_{n}$. We restate Lemma 8 below as a corollary for this special case.

Corollary 2 If $\lambda^{*}>0$, then in OPT, there exist a sequence of consecutive sensors $s_{i}, s_{i+1}, \ldots, s_{j}$ with $i \leq j$ such that they are in attached positions and one of the following three cases is true. (a) The sensor $s_{j}$ is moved to the left by the distance $\lambda^{*}, i=1$, and $y_{1}=r$. (b) The sensor $s_{i}$ is moved to the right by the distance $\lambda^{*}, j=n$, and $y_{n}=L-r$. (c) For $i \neq j$ (i.e., $i<j$ ), the sensor $s_{i}$ is moved to the right by the distance $\lambda^{*}$ and the sensor $s_{j}$ is moved to the left by the distance $\lambda^{*}$.

For any $1 \leq i<j \leq n$, we define $\lambda_{3}(i, j)$ in the same way as before, i.e., $\lambda_{3}(i, j)=\left[x_{j}-x_{i}-2 r(j-\right.$ $i)] / 2$, which corresponds to case (c) of Corollary 2, For each $1 \leq j \leq n$, define $\lambda_{1}^{\prime}(j)=x_{j}+r-2 r j$, which corresponds to case (a). Similarly, for each $1 \leq i \leq n$, define $\lambda_{2}^{\prime}(i)=L-2 r(n-i)-\left(x_{i}+r\right)$, which corresponds to case (b). We still use $\Lambda_{3}$ to denote the set of all $\lambda_{3}(i, j)$ values. Define $\Lambda_{1}^{\prime}=\left\{\lambda_{1}^{\prime}(j) \mid 1 \leq j \leq n\right\}$ and $\Lambda_{2}^{\prime}=\left\{\lambda_{2}^{\prime}(i) \mid 1 \leq i \leq n\right\}$. Let $\Lambda^{\prime}=\Lambda_{1}^{\prime} \cup \Lambda_{2}^{\prime} \cup \Lambda_{3}$. By Corollary 2, we have $\lambda^{*} \in \Lambda^{\prime}$. The following lemma is crucial to our algorithm.

Lemma 18 The optimal value $\lambda^{*}$ is the maximum value in $\Lambda^{\prime}$.

Proof: Let $\lambda^{\prime}$ be the maximum value in $\Lambda^{\prime}$. It suffices to show $\lambda^{*} \leq \lambda^{\prime}$ and $\lambda^{\prime} \leq \lambda^{*}$. Since $\lambda^{*} \in \Lambda^{\prime}$, $\lambda^{*} \leq \lambda^{\prime}$ trivially holds. Below, we focus on proving $\lambda^{\prime} \leq \lambda^{*}$.

Since $\lambda^{*}>0, \lambda^{\prime}>0$ holds. Clearly, either $\lambda^{\prime} \in \Lambda_{1}^{\prime}$, or $\lambda^{\prime} \in \Lambda_{2}^{\prime}$, or $\lambda^{\prime} \in \Lambda_{3}$. Below we analyze these three cases.

If $\lambda^{\prime} \in \Lambda_{1}^{\prime}$, then suppose $\lambda^{\prime}=\lambda_{1}^{\prime}(j)$ for some $j$. Since $\lambda^{\prime}>0$, we have $0<\lambda^{\prime}=\lambda_{1}^{\prime}(j)=$ $x_{j}+r-2 r j$, and thus $x_{j}-r>2 r(j-1)$. Since all sensors are initially on the barrier $B, x_{j} \leq L$ holds. Hence, even if all sensors $s_{1}, s_{2}, \ldots, s_{j-1}$ are somehow moved such that they are in attached positions to cover the sub-interval $[0,2 r(j-1)]$ of $B$, the sub-interval $\left[2 r(j-1), x_{j}-r\right]$ of $B$ is still not covered by any of the sensors $s_{1}, s_{2}, \ldots, s_{j-1}$. By the order preserving property, to cover the sub-interval $\left[2 r(j-1), x_{j}-r\right]$, the best way is to move $s_{j}$ to the left such that the new position of $s_{j}$ is at $2 r(j-1)+r$ (i.e., the sensors $s_{1}, s_{2}, \ldots, s_{j}$ are in attached positions), for which the moving distance of $s_{j}$ is exactly $\lambda_{1}^{\prime}(j)$. Therefore, the maximum sensor movement in any optimal solution has to be at least $\lambda_{1}^{\prime}(j)$. Thus, $\lambda^{\prime}=\lambda_{1}^{\prime}(j) \leq \lambda^{*}$.

If $\lambda^{\prime} \in \Lambda_{2}^{\prime}$, then the analysis is symmetric to the above case and we omit the details.

When $\lambda^{\prime} \in \Lambda_{3}$, the analysis has a similar spirit and we briefly discuss it. Suppose $\lambda^{\prime}=\lambda_{3}(i, j)=$ $\left[x_{j}-x_{i}-2 r(j-i)\right] / 2$ for some $i<j$. Since all sensors are initially on the barrier $B$, we have $0 \leq x_{i}<x_{j} \leq L$. Consider the sub-interval $\left[x_{i}+r, x_{j}-r\right]$ of $B$. Because $\lambda^{\prime}>0$, we have $x_{j}-x_{i}-2 r(j-i)>0$, and thus $\left(x_{j}-r\right)-\left(x_{i}+r\right)>2 r(j-i-1)$. This implies that even if we somehow move the sensors $s_{i+1}, s_{i+2}, \ldots, s_{j-1}$ such that they are in attached positions inside $\left[x_{i}+r, x_{j}-r\right]$, there are still points in $\left[x_{i}+r, x_{j}-r\right]$ that are not covered by the sensors $s_{i+1}, s_{i+2}, \ldots, s_{j-1}$. By the order preserving property, to cover the interval $\left[x_{i}+r, x_{j}-r\right]$, we have to use both $s_{i}$ and $s_{j}$ and the best way is to move $s_{i}$ to the right and move $s_{j}$ to the left by an equal distance so that all sensors $s_{i}, s_{i+1}, \ldots, s_{j}$ are in attached positions, for which the moving distances of $s_{i}$ and $s_{j}$ are both $\lambda_{3}(i, j)$ exactly. Therefore, the maximum sensor movement in any optimal solution has to be at least $\lambda_{3}(i, j)$. Thus, $\lambda^{\prime}=\lambda_{3}(i, j) \leq \lambda^{*}$.

In summary, in any case, $\lambda^{\prime} \leq \lambda^{*}$ holds. The lemma thus follows. 
Base on Lemma 18, to compute $\lambda^{*}$, we only need to find the maximum value in $\Lambda^{\prime}$, which can be easily obtained in $O\left(n^{2}\right)$ time by computing the set $\Lambda^{\prime}$ explicitly (note that $\left|\Lambda^{\prime}\right|=\Theta\left(n^{2}\right)$ ). Yet, we show below that we can find its maximum value in $O(n)$ time without computing $\Lambda^{\prime}$ explicitly.

Lemma 19 The maximum value in $\Lambda^{\prime}$ can be computed in $O(n)$ time.

Proof: Let $\lambda_{1}, \lambda_{2}$, and $\lambda_{3}$ be the maximum values in the three sets $\Lambda_{1}^{\prime}, \Lambda_{2}^{\prime}$, and $\Lambda_{3}$, respectively. It is sufficient to show how to compute $\lambda_{1}, \lambda_{2}$, and $\lambda_{3}$ in $O(n)$ time.

Both the sets $\Lambda_{1}^{\prime}$ and $\Lambda_{2}^{\prime}$ can be computed explicitly in $O(n)$ time. Thus, we can find $\lambda_{1}$ and $\lambda_{2}$ in $O(n)$ time. Below, we focus on computing $\lambda_{3}$.

Note that for each value $\lambda_{3}(i, j) \in \Lambda_{3}$ with $i<j$, we have $\lambda_{3}(i, j)=\left[x_{j}-x_{i}-2 r(j-i)\right] / 2$. For each $1 \leq t \leq n-1$, define $z_{t}=x_{t+1}-x_{t}-2 r$. Hence, $\lambda_{3}(i, j)=\left(\sum_{t=i}^{j-1} z_{t}\right) / 2$. This implies that finding the maximum value in $\Lambda_{3}$ is equivalent to finding a consecutive subsequence of $z_{1}, z_{2}, \ldots, z_{n-1}$ such that the sum of the subsequence is the maximum among all possible consecutive subsequences, which is an instance of the well studied maximum subsequence sum problem. This problem can be solved easily in $O(n)$ time. Specifically, we first compute all values $z_{1}, z_{2}, \ldots, z_{n-1}$, in $O(n)$ time. If all values are negative, then $\lambda_{3}$ is the maximum value divided by 2 . Otherwise, we let $z_{0}^{\prime}=0$, and for each $1 \leq t \leq n-1$, let $z_{t}^{\prime}=\max \left\{z_{t-1}^{\prime}, 0\right\}+z_{t}$. It is not difficult to see that $\lambda_{3}=\frac{1}{2} \cdot \max _{1 \leq t \leq n-1}\left\{z_{t}^{\prime}\right\}$. Hence, $\lambda_{3}$ can be computed in $O(n)$ time. The lemma thus follows.

After $\lambda^{*}$ is computed, we use the linear time decision algorithm for Lemma 9 to compute the destinations of all sensors such that the maximum sensor movement is at most $\lambda^{*}$.

Theorem 5 The special uniform case of the BCLS problem is solvable in $O(n)$ time.

\section{The Simple Cycle Barrier Coverage}

In this section, we discuss the simple cycle barrier coverage problem and present an $O(n)$ time algorithm for it. Mehrandish [16] gave an $O\left(n^{2}\right)$ time algorithm by somehow generalizing the $O\left(n^{2}\right)$ time algorithm [9] for the uniform BCLS.

In this problem, the target region $R$ is on the plane enclosed by a simple cycle $B$ that is the barrier we aim to cover. The sensors in $S=\left\{s_{1}, s_{2}, \ldots, s_{n}\right\}$ are initially located on $B$ and each sensor is allowed to move only on $B$ (e.g., not allowed to move inside or outside $R$ ). All sensors in $S$ have the same range $r$. Here, the distance between any two points on $B$ is not measured by their Euclidean distance in the plane but by their shortest distance along $B$. If a sensor is at a point $p$ on $B$, then it covers all points of $B$ whose distances to $p$ are at most $r$. Suppose all sensors in $S$ are initially ordered clockwise on $B$ as specified by their indices. Our goal is to move the sensors along $B$ to form a coverage of $B$ such that the maximum sensor movement is minimized.

Since $B$ is a cycle here, a sensor is said to move clockwise or counterclockwise (instead of right or left). Let $L$ be the length of $B$. Again, we assume $L \leq 2 n r$ (otherwise, it would not be possible to form a coverage of $B$ ). Since $B$ is a cycle, if $L \leq 2 r$, then every sensor by itself forms a coverage of $B$. Below, we assume $L>2 r$. Imagine that we pick a point $p_{0}$ on the interval of $B$ from $s_{n}$ clockwise to $s_{1}$ as the origin of $B$, and define the coordinate of each point $p \in B$ as the distance traversed as we move from $p_{0}$ to $p$ clockwise along $B$. Let the input coordinate of each sensor $s_{i} \in S$ be $x_{i}$. Thus, we have $0<x_{1} \leq x_{2} \leq \cdots \leq x_{n}<L$. Further, for each $1 \leq i \leq n$, we let $s_{i+n}$ denote a duplication of the sensor $s_{i}$ with a coordinate $x_{i+n}=x_{i}+L$, which actually refers to the position on $B$ with the coordinate $x_{i}$. 
Since all sensors have the same range, it is easy to see that there always exists an order preserving optimal solution $O P T$ in which the sensors are ordered clockwise along $B$ in the same order as that of their input indices. A formal proof for this is given in [16]. Again, let $\lambda^{*}$ be the optimal moving distance. We can check whether $\lambda^{*}=0$ in $O(n)$ time. Below, we assume $\lambda^{*}>0$.

Actually, our algorithm considers a set of $2 n$ sensors, $S^{\prime}=\left\{s_{1}, s_{2}, \ldots, s_{2 n}\right\}$. Specifically, the algorithm determines a consecutive sequence of sensors, $S_{i j}^{\prime}=\left\{s_{i}, s_{i+1}, \ldots, s_{j}\right\} \subset S^{\prime}$ with $1 \leq i<$ $j<i+n$, and moves the sensors of $S_{i j}^{\prime}$ to form a barrier coverage of $B$ such that the maximum sensor movement is minimized. Clearly, for each sensor $s_{k} \in S$, at most one of $s_{k}$ and its duplication $s_{k+n}$ is in $S_{i j}^{\prime}$. In this simple cycle case, the definition of attached positions of the sensors is slightly different from that in the line segment case. In this case, we call the two endpoints of the covering interval of a sensor $s_{i}$ its counterclockwise and clockwise extensions, such that when going clockwise from the counterclockwise extension to the clockwise extension, we move along the covering interval of $s_{i}$. One sensor is always in attached position by itself. Two sensors are in attached positions if the clockwise extension of one sensor is at the same position as the counterclockwise extension of the other sensor. Note that unlike in the line segment case, if two sensors $s_{i}$ and $s_{j}$ are in attached positions, say, the clockwise extension of $s_{i}$ is at the same position as the counterclockwise extension of $s_{j}$, then since the sensors are on the cycle, it is possible that the clockwise extension of $s_{j}$ is in the interior of the covering interval of $s_{i}$ (e.g., when $L<4 r$ ). Similarly, a sequence of sensors $s_{i}, s_{i+1}, \ldots, s_{j}$ (with $1 \leq i<j<i+n$ ) are in attached positions if the clockwise extension of $s_{t}$ is at the same position as the counterclockwise extension of $s_{t+1}$ for each $i \leq t \leq j-1$ (and the clockwise extension of $s_{j}$ may be in the interior of the covering interval of $s_{i}$ ). The next result is a corollary of Lemma 8 .

Corollary 3 If $\lambda^{*}>0$, then in OPT, there exist a sequence of sensors $s_{i}, s_{i+1}, \ldots, s_{j}$ in $S^{\prime}$ with $1 \leq i<j<i+n$ such that they are in attached positions and the sensor $s_{i}$ is moved clockwise by the distance $\lambda^{*}$ and the sensor $s_{j}$ is moved counterclockwise by the distance $\lambda^{*}$.

For each pair of $i$ and $j$ with $1 \leq i<j<i+n$, we define $\lambda(i, j)=\left[x_{j}-x_{i}-2 r(j-i)\right] / 2$. Let $\Lambda$ be the set of all such $\lambda(i, j)$ values. By Corollary 3 , $\lambda^{*} \in \Lambda$. The following result is similar to that of Lemma 18,

Lemma 20 The optimal value $\lambda^{*}$ is the maximum value in $\Lambda$.

Proof: The proof is very similar to that for Lemma 18 and we briefly discuss it below.

Let $\lambda^{\prime}$ be the maximum value in $\Lambda$. It is sufficient to show $\lambda^{*} \leq \lambda^{\prime}$ and $\lambda^{\prime} \leq \lambda^{*}$. Due to $\lambda^{*} \in \Lambda$, $\lambda^{*} \leq \lambda^{\prime}$ trivially holds. Hence, we focus on proving $\lambda^{\prime} \leq \lambda^{*}$. Since $\lambda^{*}>0$, we have $\lambda^{\prime}>0$.

Suppose $\lambda^{\prime}=\lambda(i, j)=\left[x_{j}-x_{i}-2 r(j-i)\right] / 2$ for some $i$ and $j$ with $1 \leq i<j<i+n$. Consider the clockwise interval $\left[x_{i}+r, x_{j}-r\right]$ on $B$, i.e., the union of the points on $B$ from $x_{i}+r$ to $x_{j}-r$ clockwise. Since $\lambda^{\prime}>0$, we have $x_{j}-x_{i}-2 r(j-i)>0$, and thus $\left(x_{j}-r\right)-\left(x_{i}+r\right)>2 r(j-i-1)$. This implies that even if we somehow move the sensors $s_{i+1}, s_{i+2}, \ldots, s_{j-1}$ such that they are in attached positions inside $\left[x_{i}+r, x_{j}-r\right]$, there are still points in $\left[x_{i}+r, x_{j}-r\right]$ that are not covered by the sensors $s_{i+1}, s_{i+2}, \ldots, s_{j-1}$. By the order preserving property, to cover the interval $\left[x_{i}+r, x_{j}-r\right]$ on $B$, the best way is to move $s_{i}$ clockwise and move $s_{j}$ counterclockwise by the same distance, for which the moving distances of $s_{i}$ and $s_{j}$ are both $\lambda(i, j)$ exactly. Therefore, the maximum sensor movement in any optimal solution has to be at least $\lambda(i, j)$. Thus, $\lambda^{\prime}=\lambda(i, j) \leq \lambda^{*}$.

The lemma thus follows. 
By using the same algorithm for Lemma 19, we can find $\lambda^{*}$ in $\Lambda$ in $O(n)$ time. With the value $\lambda^{*}$, we can then easily compute an optimal solution (i.e., compute the destinations of all sensors) in $O(n)$ time, as follows.

Suppose $\lambda^{*}=\lambda(i, j) \in \Lambda$ for some $i$ and $j$ with $1 \leq i<j<i+n$. In the case of $i>n$, we have $j>n$ and let $i=i-n$ and $j=j-n$. Thus, we still have $\lambda^{*}=\lambda(i, j)$ since $\lambda(i, j)=\lambda(i-n, j-n)$ when $i>n$ and $j>n$. Below, we assume $1 \leq i \leq n$. Note that $j>n$ is possible.

First, we move $s_{i}$ clockwise by the distance $\lambda^{*}$ and move $s_{j}$ counterclockwise by the same distance $\lambda^{*}$. Next, move all sensors $s_{i+1}, s_{i+2}, \ldots, s_{j-1}$ such that the sensors $s_{i}, s_{i+1}, \ldots, s_{j}$ are in attached positions. Since $\lambda^{*}$ is the maximum value in $\Lambda$ by Lemma 20, the above movements of the sensors $s_{i+1}, s_{i+2}, \ldots, s_{j-1}$ are at most $\lambda^{*}$. Then, starting at the sensor $s_{j+1}$, we consider the other sensors $s_{j+1}, s_{j+2}, \ldots, s_{i-1}$ of $S$ clockwise along $B$, and move them to cover the portion of $B$ that is not covered by the sensors $s_{i}, s_{i+1}, \ldots, s_{j}$. For this, we can view the remaining uncovered portion of $B$ as a line segment and apply the linear time greedy algorithm for Lemma 9 with the value $\lambda^{*}$. The overall running time is $O(n)$.

Theorem 6 The simple cycle barrier coverage problem is solvable in $O(n)$ time.

Note that the case $L<2 n r$ is also discussed in [16], where an issue of balance points appears. In the case $L \geq 2 n r$ that we consider, the issue does not exist because the entire cycle $B$ must be covered by the sensors in the optimal solution.

\section{Conclusions}

We present several algorithms on minimizing the maximum sensor movement for barrier coverage on linear domains. We present the first-known polygonal time algorithm for the problem where the barrier is a line segment and the sensors have different sensing ranges, and the algorithm runs in $O\left(n^{2} \log n\right)$ time. If the sensing ranges are the same, we give an $O(n \log n)$ time solution, and further if the sensors are initially located on the barrier segment, then the algorithm runs in $O(n)$ time. In addition, if the barrier is a simple cycle and the sensing ranges are the same, our approach can solve the problem in $O(n)$ time. Each of these results either is first-known or improves the previous best-known algorithms.

An interesting question is whether our $O\left(n^{2} \log n\right)$ time algorithm can be improved. One possible direction, as we did for the uniform case in Section 3, is to try to determine a set $\Lambda$ of candidate values such that $\lambda^{*} \in \Lambda$, and then use our decision algorithms given in Section 2.1 to find $\lambda^{*}$ in $\Lambda$. It would also be interesting to see whether our techniques can be extended to solve the non-uniform simple cycle case where the sensing ranges are different. In addition, since when all sensors are initially located on the barrier segment the uniform case can be solved faster, it is natural to ask whether our $O\left(n^{2} \log n\right)$ time algorithm for the non-uniform case can be made faster if all sensors are initially located on the barrier segment.

\section{References}

[1] I. Akyildiz, W. Su, Y. Sankarasubramaniam, and E. Cayirci. Wireless sensor networks: A survey. Computer Networks, 38(4):393-422, 2002.

[2] B. Bhattacharya, B. Burmester, Y. Hu, E. Kranakis, Q. Shi, and A. Wiese. Optimal movement of mobile sensors for barrier coverage of a planar region. Theoretical Computer Science, 410(52):5515-5528, 2009.

[3] A. Chen, S. Kumar, and T. Lai. Designing localized algorithms for barrier coverage. In Proc. of the 13th Annual ACM International Conference on Mobile Computing and Networking, pages 63-73, 2007. 
[4] D.Z. Chen, Y. Gu, J. Li, and H. Wang. Algorithms on minimizing the maximum sensor movement for barrier coverage of a linear domain. Discrete and Computational Geometry, 50:374-408, 2013.

[5] D.Z. Chen, X. Tan, H. Wang, and G. Wu. Optimal point movement for covering circular regions. arXiv:1107.1012v1, 2011.

[6] D.Z. Chen, C. Wang, and H. Wang. Representing a functional curve by curves with fewer peaks. Discrete and Computational Geometry (DCG), 46(2):334-360, 2011.

[7] R. Cole. Slowing down sorting networks to obtain faster sorting algorithms. Journal of the ACM, 34(1):200-208, 1987.

[8] R. Cole, J. Salowe, W. Steiger, and E. Szemerédi. An optimal-time algorithm for slope selection. SIAM Journal on Computing, 18(4):792-810, 1989.

[9] J. Czyzowicz, E. Kranakis, D. Krizanc, I. Lambadaris, L. Narayanan, J. Opatrny, L. Stacho, J. Urrutia, and M. Yazdani. On minimizing the maximum sensor movement for barrier coverage of a line segment. In Proc. of the 8th International Conference on Ad-Hoc, Mobile and Wireless Networks, volume 5793 of Lecture Notes in Computer Science, pages 194-212. Springer, 2009.

[10] J. Czyzowicz, E. Kranakis, D. Krizanc, I. Lambadaris, L. Narayanan, J. Opatrny, L. Stacho, J. Urrutia, and M. Yazdani. On minimizing the sum of sensor movements for barrier coverage of a line segment. In Proc. of the 9th International Conference on Ad-Hoc, Mobile and Wireless Networks, volume 6288 of Lecture Notes in Computer Science, pages 29-42. Springer, 2010.

[11] S. Hu. 'Virtual Fence' along border to be delayed. Washington Post, February 28, 2008.

[12] S. Kumar, T. Lai, and A. Arora. Barrier coverage with wireless sensors. Wireless Networks, 13(6):817-834, 2007.

[13] M. Li, X. Sun, and Y. Zhao. Minimum-cost linear coverage by sensors with adjustable ranges. In Proc. of the 6th International Conference on Wireless Algorithms, Systems, and Applications, pages 25-35, 2011.

[14] X. Li, H. Frey, N. Santoro, and I. Stojmenovic. Localized sensor self-deployment with coverage guarantee. $A C M$ SIGMOBILE Mobile Computing and Communications Review, 12(2):50-52, 2008.

[15] N. Megiddo. Applying parallel computation algorithms in the design of serial algorithms. Journal of the ACM, 30(4):852-865, 1983.

[16] M. Mehrandish. On Routing, Backbone Formation and Barrier Coverage in Wireless Ad Doc and Sensor Networks. PhD thesis, Concordia University, Montreal, Quebec, Canada, 2011.

[17] M. Mehrandish, L. Narayanan, and J. Opatrny. Minimizing the number of sensors moved on line barriers. In Proc. of IEEE Wireless Communications and Networking Conference (WCNC), pages 653-658, 2011.

[18] X. Tan and G. Wu. New algorithms for barrier coverage with mobile sensors. In Proc. of the 4th International Workshop on Frontiers in Algorithmics, volume 6213 of Lecture Notes in Computer Science, pages 327-338. Springer-Verlag, 2010.

[19] S. Yang, M. Li, and J. Wu. Scan-based movement-assisted sensor deployment methods in wireless sensor networks. IEEE Trans. Parallel Distrib. Syst., 18(8):1108-1121, 2007.

[20] Y. Zou and K. Chakrabarty. A distributed coverage and connectivity-centric technique for selecting active nodes in wireless sensor networks. IEEE Trans. Comput., 54(8):978-991, 2005. 